Article

\title{
Oncologic Benefit of Adjuvant Chemoradiation after D2 Gastrectomy: A Stepwise Hierarchical Pooled Analysis and Systematic Review
}

\author{
Chai Hong Rim ${ }^{1, *(D)}$, In-Soo Shin ${ }^{2}$, Hye Yoon Lee ${ }^{3}$, Won Sup Yoon ${ }^{1}$ and Sunmin Park ${ }^{1}$ (D) \\ 1 Department of Radiation Oncology, Ansan Hospital, Korea University Medical College, \\ Gyeonggido 15355, Korea; irionyws@korea.ac.kr (W.S.Y.); sunmini815@gmail.com (S.P.) \\ 2 Graduate school of Education, Dongguk University, Seoul 04620, Korea; s9065031@dongguk.edu \\ 3 Department of General Surgery, Ansan Hospital, Korea University Medical College, Ansan, \\ Gyeonggido 15355, Korea; heygemma@korea.ac.kr \\ * Correspondence: crusion@korea.ac.kr; Tel.: +82-31-412-6850; Fax: +82-31-412-6851
}

Received: 14 July 2020; Accepted: 30 July 2020; Published: 31 July 2020

\begin{abstract}
Our study aimed to evaluate the benefits of chemoradiotherapy (CRT) after D2 gastrectomy, as compared to adjuvant chemotherapy, alone. PubMed, MEDLINE, Embase, and the Cochrane Library were systematically searched. We applied stepwise analyses that enabled the evaluation of data from randomized controlled trials (RCTs), balanced studies, and all studies separately and in a hierarchical manner. Thirteen controlled studies, including six RCTs involving 2603 patients, were included. Overall pooled analysis revealed a disease-free survival benefit of CRT (odds ratio (OR): 1.264, $p=0.053$ ), which was more evident in the subgroup analysis of RCTs (OR: 1.440, $p=0.006$ ) and balanced studies (OR: 1.417, $p<0.001$ ). Overall survival was insignificantly different in the overall pooled analysis (OR: 1.124, $p=0.347$ ). However, the difference was marginally significant in the subgroup analysis of balanced studies (OR: 1.279, $p=0.055$ ) and significant in the subgroup analysis of studies involving stage $\geq$ III patients only (OR: 1.663, $p=0.005$ ). Locoregional recurrence (LRR) reduction was noted in the overall pooled analysis (OR: $0.559, p=0.012$; pooled rate: $11.3 \% \mathrm{vs}$. $18.1 \%$ ) and was more robust in the subgroup analyses. Grade $\geq 3$ leukopenia was higher in the CRT arm (OR: 1.387, $p=0.004$; pooled rate: $26.4 \%$ vs. $15.7 \%$ ). CRT after D2 gastrectomy should be applied for patients with high risk of LRR (e.g., stage $\geq$ III), along with efforts to reduce leukopenia.
\end{abstract}

Keywords: D2 gastrectomy; stomach cancer; gastric cancer; chemoradiotherapy; radiation therapy

\section{Introduction}

Gastric cancer is responsible for up to 800,000 deaths annually and is the third leading cause of cancer-related death [1]. The mainstay curative modality for gastric cancer is surgical resection. Except for early-stage cases that are amenable to endoscopic resection (e.g., well-differentiated tumors $<2 \mathrm{~cm}$ in size), gastrectomy with D2 lymphatic dissection (D2 gastrectomy) is commonly applied, specifically in Asian countries [2-6]. Adjuvant chemotherapy (CT) was previously found to benefit western patients, in whom D2 gastrectomy is less commonly performed [7,8]. Recent landmark randomized controlled trials (RCTs) confirmed the oncologic benefit of S-1 or capecitabine plus oxaliplatin for Asian patients; hence, adjuvant CT is widely administered to these patients (including those who underwent $\mathrm{D} 2$ gastrectomy) $[9,10]$.

Although the Intergroup-0116 trial revealed the benefit of adjuvant chemoradiotherapy (CRT) [11], it was heavily criticized for its insufficient extent of surgery, as fewer than $10 \%$ of patients underwent D2 gastrectomy. Therefore, a large tertiary center in South Korea conducted an RCT that compared the benefit of CRT to that of CT in patients who underwent D2 gastrectomy. This "Adjuvant 
Chemoradiation Therapy in Stomach Cancer" (ARTIST) trial failed to meet its primary endpoint of disease-free survival (DFS, $p=0.0862$ ) [12]. Therefore, adjuvant CRT is generally not recommended after D2 gastrectomy in major clinical guidelines except for high-risk subgroups with R1 resection or remnant disease [2-4,13-15].

Locoregional recurrence (LRR) remains a burden even after D2 gastrectomy. In a previous large surgical series of up to 1500 patients who underwent D2 gastrectomy [16], grossly one-quarter experienced recurrences, approximately one-third (32.5\%) of which were locoregional. The survival period for patients with LRR was only 1.5 months longer than that for patients with hematogenous metastases. Although the primary endpoint was not reached in the ARTIST trial, LRR was significantly reduced ( $7 \%$ vs. $13 \%, p=0.0033)$ and DFS was extended following CRT in the subgroup of patients with lymphatic metastases [17]. Several other clinical trials also revealed LRR and DFS benefits for CRT, which reflected real-world clinical experiences and practices [18-21]. Furthermore, recent technological advancements in radiation therapy have gradually lowered the risk of possible complications by reducing bystander irradiation to the organs-at-risk [22-24].

Our study aimed to integrate and analyze data from controlled trials that compared CRT and CT for patients with gastric cancer who underwent D2 gastrectomy and to thereby evaluate the practical role of CRT using real-world studies and RCTs.

\section{Results}

Thirteen studies [17-21,25-32] involving 2603 patients with gastric cancer who underwent D2 resection were included (Figure 1). Six studies were RCTs $[17,18,21,26,29,30]$ and seven were non-RCTs [19,20,25,27,28,31,32]; nine studies were considered balanced between their two arms [17-21, 29-32]. The studies' quality scores are shown in Table S1; all studies included patients who achieved $\mathrm{R} 0$ resection after $\mathrm{D} 2$ gastrectomy except one in which approximately one-quarter of the patients had undergone R1 resection [27]. The studies' backgrounds and clinical information are shown in Tables 1 and 2, respectively.

The pooled odds ratio (OR) for DFS was 1.264 (95\% confidence interval (CI): 0.997-1.603, $p=0.053$ ) favoring the CRT arm with a medium degree of heterogeneity ( $\left.p=0.1, \mathrm{I}^{2}=37.5 \%\right)$. In the subgroup analyses of RCTs alone, the pooled OR increased to 1.440 (95\% CI: 1.110-1.867, $p=0.006$ ), and heterogeneity was significantly diminished $\left(p=0.964, \mathrm{I}^{2}=\sim 0.0 \%\right)$. Subgroup analysis of only the balanced studies showed similar results, with an OR of 1.417 (95\% CI: 1.171-1.715, $p<0.001$ ) and very low heterogeneity $\left(p=0.61, \mathrm{I}^{2}=\sim 0.0 \%\right)$. In single-arm analyses, the 3-year DFS rates were $62.5 \%$ (95\% CI: 54.6-69.8) and 57.1\% (95\% CI: 46.1-67.5) in the CRT and CT arms, respectively; the corresponding 5-year DFS rates were 53.3\% (95\% CI: 40.9-56.4) and 40.9\% (95\% CI: $27.3-56.0)$, respectively. The heterogeneity among studies in the single-arm analyses was very high and significant. 

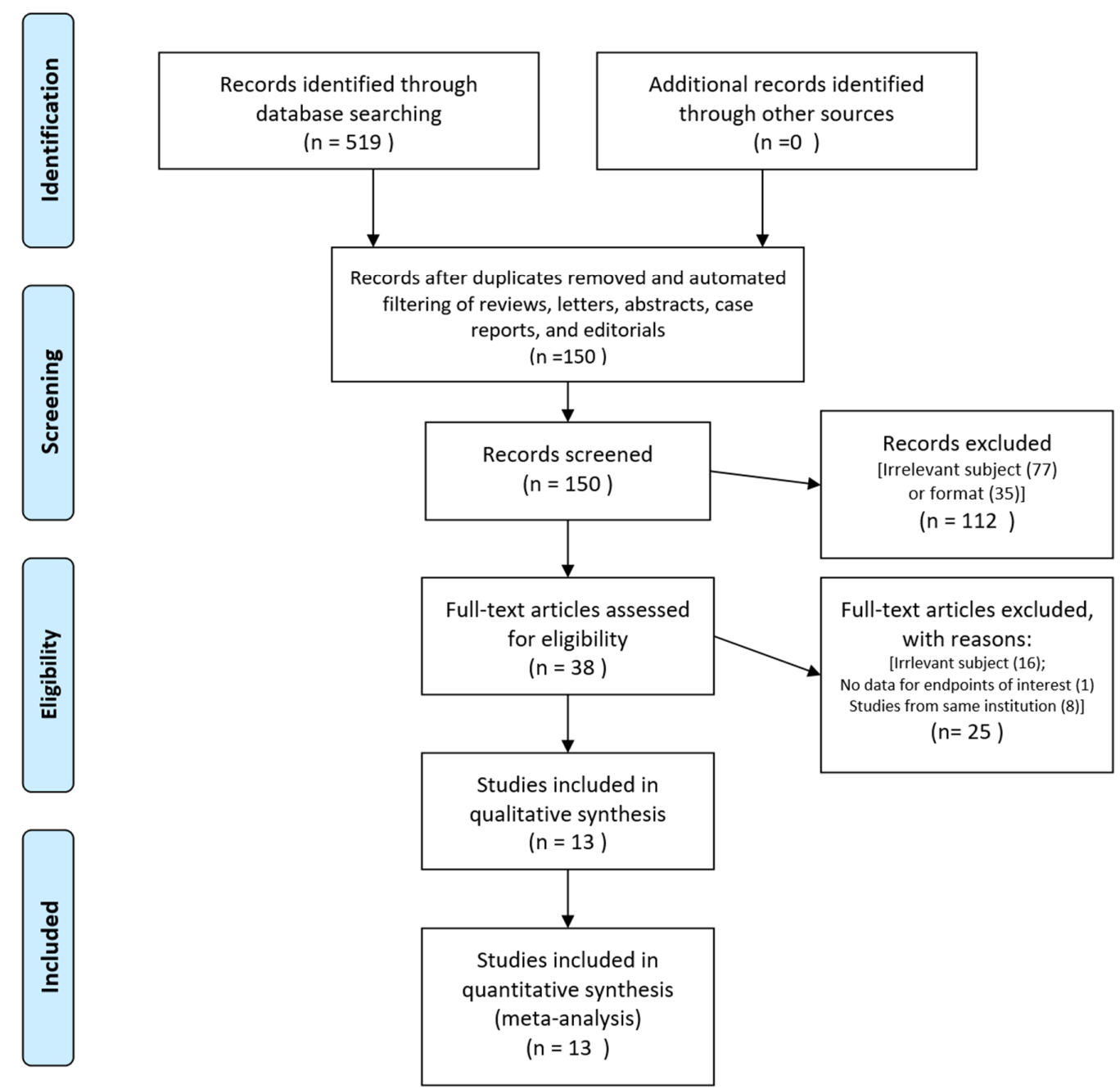

Figure 1. Flowchart of the study selection process. 
Table 1. Background information of patients in the included studies.

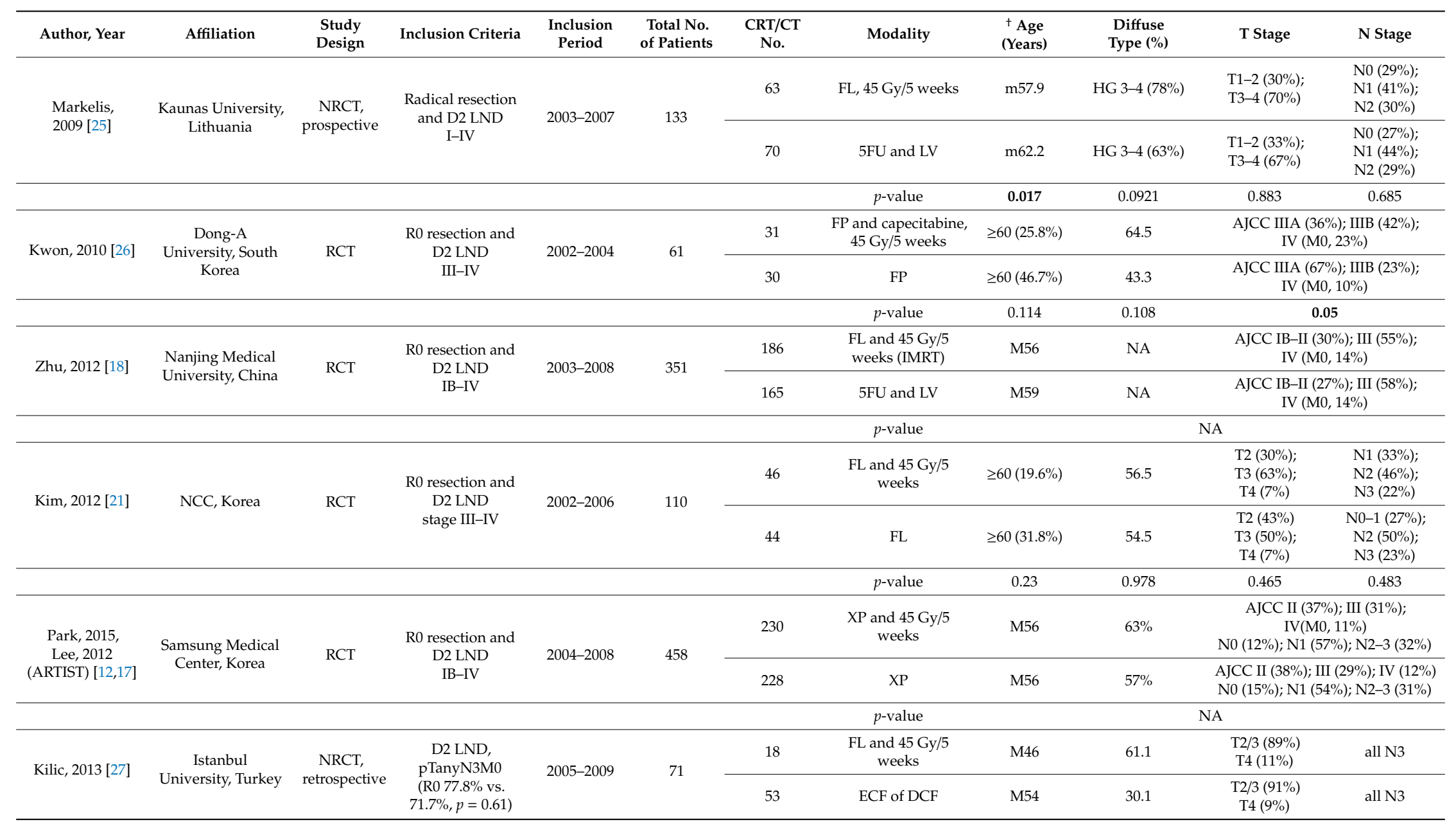


Table 1. Cont.

\begin{tabular}{|c|c|c|c|c|c|c|c|c|c|c|c|}
\hline Author, Year & Affiliation & $\begin{array}{c}\text { Study } \\
\text { Design }\end{array}$ & Inclusion Criteria & $\begin{array}{c}\text { Inclusion } \\
\text { Period }\end{array}$ & $\begin{array}{c}\text { Total No. } \\
\text { of Patients }\end{array}$ & $\begin{array}{l}\text { CRT/CT } \\
\text { No. }\end{array}$ & Modality & $\begin{array}{l}{ }^{\dagger} \text { Age } \\
\text { (Years) }\end{array}$ & $\begin{array}{c}\text { Diffuse } \\
\text { Type (\%) }\end{array}$ & T Stage & N Stage \\
\hline & & & & & & & $p$-value & 0.1 & 0.02 & 0.72 & \\
\hline \multirow[b]{2}{*}{ Lee, 2014 [28] } & \multirow[b]{2}{*}{$\begin{array}{l}\text { Samsung Medical } \\
\text { Center, Korea }\end{array}$} & \multirow{2}{*}{$\begin{array}{l}\mathrm{NRCT}, \\
\text { retrospective }\end{array}$} & \multirow{2}{*}{$\begin{array}{l}\text { R0 resection and } \\
\text { D2 LND } \\
\text { IB-IV }\end{array}$} & \multirow[b]{2}{*}{ 2008-2009 } & \multirow[b]{2}{*}{405} & 244 & $\begin{array}{c}\text { FL and } 45 \mathrm{~Gy} / 5 \\
\text { weeks }\end{array}$ & M53 & $\begin{array}{l}\text { Tubular }(68.4 \%) \\
\text { signet ring } \\
\text { cell }(22.1 \%)\end{array}$ & $\begin{array}{l}\text { T1-2 (71\%) } \\
\text { T3-4 }(29 \%)\end{array}$ & $\begin{array}{l}\text { N0-2 }(56 \%) \\
\text { N3a }(27 \%) \\
\text { N3b }(17 \%) \\
\end{array}$ \\
\hline & & & & & & 161 & $S-1$ & M57 & $\begin{array}{c}\text { Tubular }(67.1 \%) \\
\text { signet ring } \\
\text { cell }(21.1 \%)\end{array}$ & $\begin{array}{l}\text { T1-2 (82\%) } \\
\text { T3-4 (18\%) }\end{array}$ & $\begin{array}{c}\text { N0-2 (78\%, } \\
\text { N3a }(17 \%) \\
\text { N3b }(5 \%)\end{array}$ \\
\hline & & & & & & & $p$-value & 0.001 & 0.234 & 0.02 & $<0.001$ \\
\hline \multirow[t]{2}{*}{ Cao, 2015 [29] } & \multirow{2}{*}{$\begin{array}{c}\text { Xinwen Mining } \\
\text { Group Central } \\
\text { Hospital, China }\end{array}$} & \multirow[t]{2}{*}{ RCT } & \multirow{2}{*}{$\begin{array}{l}\text { R0 resection and } \\
\text { D2 LND } \\
\text { stage III-IV }\end{array}$} & \multirow[t]{2}{*}{ 2008-2010 } & \multirow[t]{2}{*}{50} & 25 & $\begin{array}{c}\text { FP and capecitabine } \\
\text { CCRT } \\
\end{array}$ & $\geq 60(40 \%)$ & LD $(68 \%)$ & \multicolumn{2}{|c|}{ AJCC IIIA (32\%); IIIB (40\%) } \\
\hline & & & & & & 25 & FP & $\geq 60(44 \%)$ & LD (60\%) & AJCC IIIA & ); IIIB (32\%) \\
\hline & & & & & & & $p$-value & NS & NS & \multicolumn{2}{|c|}{ NS } \\
\hline \multirow{2}{*}{ Turanli, 2015 [20] } & \multirow{2}{*}{$\begin{array}{l}\text { Ankara Oncology } \\
\text { Education and } \\
\text { Research Hospital, } \\
\text { Turkey }\end{array}$} & \multirow{2}{*}{$\begin{array}{l}\mathrm{NRCT}, \\
\text { retrospective }\end{array}$} & \multirow{2}{*}{$\begin{array}{l}\text { R0 resection and } \\
\text { D2 LND } \\
\text { III }\end{array}$} & \multirow{2}{*}{ 2004-2009 } & \multirow{2}{*}{92} & 71 & $\begin{array}{l}\text { FL and } 45 \mathrm{~Gy} / 5 \\
\text { weeks }\end{array}$ & $\mathrm{m} 57.5$ & 29.6 & $\begin{array}{l}\text { T3 }(7 \%) \\
\text { T4 }(89 \%)\end{array}$ & $\begin{array}{l}\text { N1 }(18 \%) ; \\
\text { N2 }(40 \%) \\
\text { N3 }(42 \%)\end{array}$ \\
\hline & & & & & & 21 & FL or ECF & $\mathrm{m} 57.5$ & 38.1 & $\begin{array}{l}\text { T3 }(14 \%) \\
\text { T4 }(81 \%)\end{array}$ & $\begin{array}{l}\text { N1 }(14 \%) ; \\
\text { N2 }(38 \%) ; \\
\text { N3 }(47 \%)\end{array}$ \\
\hline & & & & & & & $p$-value & 0.98 & 0.46 & 0.48 & 0.87 \\
\hline \multirow{2}{*}{$\mathrm{Yu}, 2016$ [30] } & \multirow{2}{*}{$\begin{array}{l}\text { Anhui University, } \\
\text { China }\end{array}$} & \multirow{2}{*}{ RCT } & \multirow{2}{*}{$\begin{array}{l}\text { R0 resection and } \\
\text { D2 LND } \\
\text { stage II-III }\end{array}$} & \multirow{2}{*}{ 2010-2011 } & \multirow{2}{*}{79} & 40 & $\begin{array}{c}\text { Capecitabine } \\
\text { CCRT ( } 45 \text { Gy) } \\
\text { followed by XELOX }\end{array}$ & $\geq 60(57.5 \%)$ & $\begin{array}{l}\text { HG3-4 } \\
(57.5 \%)\end{array}$ & \multicolumn{2}{|c|}{$\begin{array}{c}\text { AJCC II (40\%) III (60\%) } \\
\text { N0 ( } 29 \%)\end{array}$} \\
\hline & & & & & & 39 & XELOX & $\geq 60(48.7 \%)$ & HG3-4 (64.1\%) & \multicolumn{2}{|c|}{$\begin{array}{c}\text { AJCC II (36\%) III (64\%) } \\
\text { N0 (31\%) }\end{array}$} \\
\hline & & & & & & & $p$-value & 0.434 & 0.548 & \multicolumn{2}{|c|}{0.707} \\
\hline \multirow[t]{2}{*}{ Peng, 2016 [31] } & \multirow{2}{*}{$\begin{array}{l}\text { Wuhan University, } \\
\text { China }\end{array}$} & \multirow[t]{2}{*}{$\begin{array}{l}\mathrm{NRCT}, \\
\text { retrospective }\end{array}$} & \multirow{2}{*}{$\begin{array}{l}\text { R0 resection and } \\
\text { D2 LND } \\
\text { stage IIA-IIIC }\end{array}$} & \multirow[t]{2}{*}{ 2004-2012 } & 337 & 124 & $\begin{array}{c}\text { FOLFOX or XELOX } \\
\text { or capecitabine and } \\
45 \mathrm{~Gy} / 5 \text { weeks }\end{array}$ & M54 & HG 3-4 (78.2\%) & $\begin{array}{r}\text { AJCC II (3 } \\
\text { IIIB (17 }\end{array}$ & $\begin{array}{l}\text { IIIA (20\%); } \\
\text { IC (27\%) }\end{array}$ \\
\hline & & & & & & 213 & $\begin{array}{l}\text { FOLFOX or XELOX } \\
\text { or capecitabine }\end{array}$ & M56 & HG 3-4 (75.5\%) & $\begin{array}{r}\text { AJCC II (3 } \\
\text { IIIB }(20 \\
\end{array}$ & $\begin{array}{l}\text { IIIA ( }(23 \%) \text {; } \\
\text { IC }(27 \%)\end{array}$ \\
\hline & & & & & & & $p$-value & 0.247 & 0.128 & & \\
\hline $\mathrm{Li}, 2017[32]$ & $\begin{array}{c}\text { Fudan University, } \\
\text { China }\end{array}$ & $\begin{array}{l}\mathrm{NRCT} \text {, } \\
\text { retrospective }\end{array}$ & $\begin{array}{l}\text { R0 resection and } \\
\text { D2 LND }\end{array}$ & 2005-2010 & 186 & 93 & $\begin{array}{c}5 \mathrm{FU} \text { or capecitabine } \\
\text { or tegafur and } \\
45-50 \mathrm{~Gy} / \\
5-6 \text { weeks (3D-CRT } \\
\text { or IMRT) } \\
\end{array}$ & m54 & LD (15.1\%) & $\begin{array}{c}\text { T1-2 (17\%) } \\
\text { T3 (25\%) } \\
\text { T4 (58\%) }\end{array}$ & $\begin{array}{l}\text { N0 (9\%); } \\
\text { N1 (17\%); } \\
\text { N2 (23\%); } \\
\text { N3 (52\%) }\end{array}$ \\
\hline & & & IB-IIIC & & & 93 & $5 \mathrm{FU}$ based regimen & $\mathrm{m} 57$ & LD $(19.4 \%)$ & $\begin{array}{c}\text { T1-2 }(14 \%) \\
\text { T3 }(19 \%) \\
\text { T4 }(67 \%)\end{array}$ & $\begin{array}{l}\text { N0 }(11 \%) ; \\
\text { N1 (22\%); } \\
\text { N2 (24\%); } \\
\text { N3 }(44 \%)\end{array}$ \\
\hline
\end{tabular}


Table 1. Cont

\begin{tabular}{|c|c|c|c|c|c|c|c|c|c|c|c|}
\hline Author, Year & Affiliation & $\begin{array}{c}\text { Study } \\
\text { Design }\end{array}$ & Inclusion Criteria & $\begin{array}{c}\text { Inclusion } \\
\text { Period }\end{array}$ & $\begin{array}{c}\text { Total No. } \\
\text { of Patients }\end{array}$ & $\begin{array}{c}\text { CRT/CT } \\
\text { No. }\end{array}$ & Modality & $\begin{array}{l}{ }^{\dagger} \text { Age } \\
\text { (Years) }\end{array}$ & $\begin{array}{c}\text { Diffuse } \\
\text { Type (\%) }\end{array}$ & T Stage & N Stage \\
\hline & & & & & & & $p$-value & 0.61 & 0.43 & 0.37 & 0.76 \\
\hline \multirow[t]{3}{*}{ Ma, 2019 [19] } & \multirow[t]{2}{*}{$\begin{array}{l}\text { Fudan University, } \\
\text { China }\end{array}$} & \multirow[t]{2}{*}{$\begin{array}{l}\text { NRCT, } \\
\text { retrospective, } \\
\text { PSM }\end{array}$} & \multirow[t]{2}{*}{$\begin{array}{l}\text { R0 resection and } \\
\text { D2 LND } \\
\text { stage III }\end{array}$} & \multirow[t]{3}{*}{ 2009-2014 } & \multirow[t]{3}{*}{270} & 135 & $\begin{array}{c}\text { Same regimen and } \\
45 \text { Gy/ } 5 \text { weeks, } \\
5-10 \text { Gy } \\
\text { boost (3D-CRT or } \\
\text { IMRT) }\end{array}$ & m54.3 & NA & $\begin{array}{l}\text { T2 }(6 \%) \\
\text { T3 (16\%) } \\
\text { T4 }(79 \%)\end{array}$ & $\begin{array}{l}\text { N0-2 (31\%) } \\
\text { N3a }(45 \%) \\
\text { N3b (24\%) }\end{array}$ \\
\hline & & & & & & 135 & $\begin{array}{l}\text { 5FU or capecitabine } \\
\text { or ECF, modified } \\
\text { DCF regimen }\end{array}$ & m54.7 & NA & $\begin{array}{l}\text { T2 }(3 \%) \\
\text { T3 }(21 \%) \\
\text { T4 }(76 \%)\end{array}$ & $\begin{array}{c}\text { N0-2 }(30 \%) \\
\text { N3a }(44 \%) \\
\text { N3b }(25 \%)\end{array}$ \\
\hline & & & & & & & $p$-value & 0.74 & & 0.305 & 0.081 \\
\hline
\end{tabular}

Abbreviations: CRT, chemoradiation; CCRT, concurrent chemoradiation; CT, chemotherapy; RCT, randomized controlled trial; LND, lymph node dissection; 5FU, 5-fluorouracil; FL, 5-fluorouracil and leucovorin; FP, 5-fluorouracil plus cisplatin; IMRT, intensity-modulated radiotherapy; NA, not assessed; NCC, National Cancer Center; XP, capecitabine and cisplatin; AJCC, American Joint Committee on Cancer; ECF, epirubicin, cisplatin, fluorouracil; DCF, docetaxel, cisplatin, fluorouracil; LD, low differentiation; NRCT, non-randomized controlled trials; XELOX, capecitabine and oxaliplatin; HG, high-grade; PSM, propensity score matching. ${ }^{+}$Upper case M denotes the median, and lower case m denotes the mean. Statistically significant $p$-values marked in bold font. 
Table 2. Clinical outcomes of patients in the included studies.

\begin{tabular}{|c|c|c|c|c|c|c|c|c|c|c|c|}
\hline Author, Year & $\begin{array}{l}\text { CRT/CT } \\
\text { No. }\end{array}$ & $\begin{array}{c}\text { Median } \\
\text { Follow-Up } \\
\text { (Months, Range) }\end{array}$ & $\begin{array}{l}\text { Median } \\
\text { DFS }\end{array}$ & 3-Year DFS & 5-Year DFS & $\begin{array}{l}\text { Median } \\
\text { OS (Months) }\end{array}$ & 3-Year OS & 5-Year OS & $\begin{array}{l}\text { Prognosticators } \\
\text { ( } p \text {-Value) }\end{array}$ & Pattern of Failure & $\begin{array}{l}\text { Grade 3-4 Toxicities } \\
\text { (CRT vs. CT) }\end{array}$ \\
\hline \multirow{2}{*}{$\begin{array}{l}\text { Markelis, } \\
2009[25]\end{array}$} & 63 & & & & & & $52.4 \%$ & & & & \multirow{2}{*}{$\begin{array}{l}\text { Overall incidence: } 44.4 \% \text { vs. } \\
\quad 7.1 \%(p<0.05)\end{array}$} \\
\hline & 70 & & & & & & $57.1 \%$ & & & & \\
\hline & & & & & & & 0.039 & & & & \\
\hline \multirow{2}{*}{ Kwon, 2010 [26] } & 31 & \multirow{2}{*}{$\begin{array}{c}77.2 \\
(24-92.8)\end{array}$} & & $80.0 \%$ & $76.7 \%$ & & $80.6 \%$ & $70.1 \%$ & & \multirow{2}{*}{$\begin{array}{c}\text { Overall LRR } 12.9 \% \text { vs. } \\
23.3 \%(p=0.335) ; \mathrm{DM} \\
9.7 \% \text { vs. } 23.3 \%(p=0.335)\end{array}$} & \multirow{2}{*}{$\begin{array}{c}\text { Neutropenia ( } 48.4 \% \text { vs. } \\
\text { 16.7\%); anemia }(12.9 \% \text { vs. } \\
16.7 \%) ; \mathrm{N} / \mathrm{V}(6.5 \% \text { vs. } 13.3 \%)\end{array}$} \\
\hline & 30 & & & $75.2 \%$ & $59.1 \%$ & & $76.7 \%$ & $70.0 \%$ & & & \\
\hline & & & & 0.887 & 0.222 & & & 0.814 & & & \\
\hline \multirow{2}{*}{ Zhu, 2012 [18] } & 186 & \multirow{2}{*}{42.5} & 50 & $57.5 \%$ & $45.2 \%$ & 54.0 & $59.7 \%$ & $48.4 \%$ & \multirow{2}{*}{$\begin{array}{l}\text { UVA, OS: Stage } \\
(p<0.001) ; \mathrm{LN}+ \\
(p=0.001)\end{array}$} & \multirow{2}{*}{$\begin{array}{c}5 \text {-yr LRR } 15.6 \% \text { vs. } 24.2 \% \\
\text { (CRT vs. CT, } p=0.042) ; \\
\text { 5-yr DM } 24.2 \% \text { vs. } 26.7 \% \\
(p=0.595)\end{array}$} & \multirow{2}{*}{$\begin{array}{l}\text { Leukopenia ( } 7.5 \% \text { vs. } 7.3 \%) ; \\
\text { nausea }(2.7 \% \text { vs. } 0 \%) ; \\
\text { vomiting }(1.6 \% \text { vs. } 0 \%)\end{array}$} \\
\hline & 165 & & 32 & $46.7 \%$ & $35.8 \%$ & 38.0 & $50.3 \%$ & $41.4 \%$ & & & \\
\hline & & & & 0.029 & & & 0.122 & & & & \\
\hline \multirow{3}{*}{ Kim, 2012 [21] } & 46 & \multirow{2}{*}{86.7} & & $67.4 \%$ & $60.9 \%$ & & & $65.2 \%$ & & \multirow{2}{*}{$\begin{array}{c}\text { Overall LRR } 10.9 \% \text { vs. } \\
34.1 \% \text { (CRT vs. CT, } \\
p=0.006) ; \text { Overall DM } \\
32.6 \% \text { vs. } 47.7 \text { (CRT vs. } \\
\text { CT, } p=0.288 \text { ) }\end{array}$} & \multirow{2}{*}{$\begin{array}{c}\text { Hematologic }(19.6 \% \text { vs. } 25 \% \\
p=\text { NS); GI toxicity }(17.4 \% \text { vs. } \\
11.4 \%, p=\text { NS). }\end{array}$} \\
\hline & 44 & & & $59.1 \%$ & $50.0 \%$ & & & $54.6 \%$ & & & \\
\hline & & & & 0.246 & & & 0.67 & & & & \\
\hline \multirow{3}{*}{$\begin{array}{l}\text { Park, } 2015 \\
\text { Lee, 2012 } \\
\text { (ARTIST) [12,17] }\end{array}$} & 230 & \multirow{2}{*}{7 years } & & $78.2 \%$ & $73.9 \%$ & & $80.0 \%$ & $75.0 \%$ & \multirow{2}{*}{$\begin{array}{l}\text { MVA, OS: Stage } \\
(p<0.01) ; \text { Lauren } \\
\text { classification } \\
(p=0.03) ; \text { LNR } \\
(p<0.01)\end{array}$} & \multirow{2}{*}{$\begin{array}{l}\text { Overall LRR } 7 \% \text { vs. } 13 \% \\
\text { (CRT vs. CT, } p=0.0033) ; \\
\text { DM } 24 \% \text { vs. } 27 \% \\
\quad(p=0.5568)\end{array}$} & \multirow{2}{*}{$\begin{array}{c}\text { Neutropenia ( } 48.4 \% \text { vs. } \\
40.7 \%) ; \text { Nausea }(12.3 \% \text { vs. } \\
12.4 \%) ; \text { Vomiting ( } 3.5 \% \text { vs. } \\
3.1 \%) ; \text { One } 5 \text { complication in } \\
\text { each arm }\end{array}$} \\
\hline & 228 & & & $74.2 \%$ & $67.1 \%$ & & $83.7 \%$ & $73.0 \%$ & & & \\
\hline & & & & 0.0862 & & & 0.527 & & & & \\
\hline \multirow{2}{*}{ Kilic, 2013 [27] } & 18 & \multirow{2}{*}{$\begin{array}{c}13.8 \\
(6.2-74.1)\end{array}$} & 15.2 & & & 34.2 & $38.8 \%$ & & \multirow{2}{*}{$\begin{array}{l}\text { MVA, DFS: LNR } \\
\quad(p=0.04)\end{array}$} & \multirow{2}{*}{$\begin{array}{c}\text { Overall LRR } 33.3 \% \text { vs. } \\
15.1 \% \text { (CRT vs. CT, } \\
p=0.63 \text { ); DM } 22.2 \% \text { vs. } \\
41.5 \% \text { (CRT vs. CT) }\end{array}$} & \multirow{2}{*}{$\begin{array}{l}\text { No toxicity related death in } \\
\text { both groups }\end{array}$} \\
\hline & 53 & & 12.5 & & & 26.8 & $39.6 \%$ & & & & \\
\hline & & & & 0.56 & & & 0.74 & & & & \\
\hline \multirow{2}{*}{ Lee, 2014 [28] } & 244 & \multirow{2}{*}{$\begin{array}{c}49 \\
(3.0-62.0)\end{array}$} & & $73.0 \%$ & & & $79.8 \%$ & & \multirow{2}{*}{$\begin{array}{l}\text { MVA, DFS: Stage } \\
\quad(<0.001) ; \text { Age } \\
(p=0.006)\end{array}$} & & \multirow{2}{*}{$\begin{array}{c}\text { Neutropenia } 40.2 \% \text { vs. } 8.7 \% \\
(p<0.001) ; \text { all neutropenia } \\
\text { was transient. Nausea } 5.7 \% \\
\text { vs. } 0 \%(p=0.002) ; \text { vomiting } \\
2.5 \% \text { vs. } 0 \%(p=0.085)\end{array}$} \\
\hline & 161 & & & $81.4 \%$ & & & $87.7 \%$ & & & & \\
\hline & & & & 0.035 & & & & & & & \\
\hline \multirow{2}{*}{ Cao, 2015 [29] } & 25 & \multirow[b]{2}{*}{3 years } & & $76.0 \%$ & $60.0 \%$ & & & $64.0 \%$ & & & Neutropenia $48 \%$ vs. $32 \%$ \\
\hline & 25 & & & $64.0 \%$ & $52.0 \%$ & & & $60.0 \%$ & & & $\begin{array}{c}(p=0.032) ; \text { diarrhea } 8 \% \text { vs. } \\
0 \%(p=0.025)\end{array}$ \\
\hline
\end{tabular}


Table 2. Cont.

\begin{tabular}{|c|c|c|c|c|c|c|c|c|c|c|c|}
\hline Author, Year & $\begin{array}{l}\text { CRT/CT } \\
\text { No. }\end{array}$ & $\begin{array}{c}\text { Median } \\
\text { Follow-Up } \\
\text { (Months, Range) }\end{array}$ & $\begin{array}{c}\text { Median } \\
\text { DFS }\end{array}$ & 3-Year DFS & 5-Year DFS & $\begin{array}{l}\text { Median } \\
\text { OS (Months) }\end{array}$ & 3-Year OS & 5-Year OS & $\begin{array}{l}\text { Prognosticators } \\
(p \text {-Value })\end{array}$ & Pattern of Failure & $\begin{array}{l}\text { Grade 3-4 Toxicities } \\
\text { (CRT vs. CT) }\end{array}$ \\
\hline & & & & 0.112 & 0.231 & & & 0.324 & & & \\
\hline \multirow{3}{*}{ Turanli, 2015 [20] } & 71 & \multirow{2}{*}{$30(8-112)$} & & $42.2 \%$ & $32.9 \%$ & 32.0 & $43.6 \%$ & $34.4 \%$ & & \multirow{2}{*}{$\begin{array}{c}\text { Overall LRR } 15.7 \% \text { vs. } \\
14.3 \% \text { (CRT vs. CT, } \\
p=0.089) ; \mathrm{DM} 45.1 \% \text { vs. } \\
57.1 \%(p=0.42)\end{array}$} & \\
\hline & 21 & & & $42.8 \%$ & $24.1 \%$ & 29.0 & $42.8 \%$ & $23.8 \%$ & & & \\
\hline & & & & 0.8 & & & 0.74 & & & & \\
\hline \multirow{2}{*}{$\mathrm{Yu}, 2016$ [30] } & 40 & \multirow{2}{*}{34} & & $42.5 \%$ & & & $52.5 \%$ & & \multirow{2}{*}{$\begin{array}{c}\text { MVA, OS: ECOG } \\
(0.016) ; \mathrm{LN}+(0.035)\end{array}$} & \multirow{2}{*}{\multicolumn{2}{|c|}{$\begin{array}{c}\text { Leukopenia }(10 \% \text { vs. } 7.7 \%, \\
p=0.253) ; \mathrm{N} / \mathrm{V}(17.5 \% \text { vs. } 12.8 \%, \\
p=0.043)\end{array}$}} \\
\hline & 39 & & & $28.2 \%$ & & & $38.5 \%$ & & & & \\
\hline & & & & 0.238 & & & 0.235 & & & & \\
\hline \multirow{2}{*}{ Peng, 2016 [31] } & 124 & \multirow{2}{*}{$\begin{array}{c}41.1 \\
(14-111.1)\end{array}$} & 40.7 & $55.6 \%$ & $38.7 \%$ & 51.0 & $41.4 \%$ & $45.6 \%$ & & \multirow{2}{*}{\multicolumn{2}{|c|}{$\begin{array}{c}\text { Overall incidence: } 36.3 \% \text { vs. } \\
31.0 \% \text { ( } p=0.338) \\
\text { m/c Cx: Leukopenia or } \\
\text { neutropenia }(21.7 \% \text { vs. } 14.6 \% \\
p=0.09) ; \text { nausea }(10.4 \% \text { vs. } 8.4 \%) \\
\text { vomiting }(9.6 \% \text { vs. } 7.5 \%)\end{array}$}} \\
\hline & 213 & & 31.2 & $43.7 \%$ & $31.1 \%$ & 48.6 & $34.7 \%$ & $37.3 \%$ & & & \\
\hline & & & 0.112 & & & 0.3 & & 0.132 & & & \\
\hline \multirow{3}{*}{ Li, 2017 [32] } & 93 & \multirow{2}{*}{$\begin{array}{l}\text { CRT } 28(5-62) \\
\text { CT } 43(2-63)\end{array}$} & & $57.0 \%$ & & & $72.8 \%$ & & & \multirow{2}{*}{$\begin{array}{c}\text { Overall LRR } 3.2 \% \text { vs. } 4.3 \% \\
(p=0.76) ; \text { DM } 12.9 \% \text { vs. } \\
23.7 \%(p=0.18)\end{array}$} & \multirow{2}{*}{$\begin{array}{c}\text { Overall incidence: } 38.7 \% \text { vs. } \\
18.3 \% \text { ( } p=0.002) ; \text { leukopenia } \\
30.1 \% \text { vs. } 10.8 \% \text {, Nausea }(10.8 \% \\
\text { vs. } 5.4 \%) \text {; vomiting } \\
\text { (5.4\% vs. } 5.4 \%)\end{array}$} \\
\hline & 93 & & & $62.0 \%$ & & & $77.4 \%$ & & & & \\
\hline & & & & 0.3 & & & 0.23 & & & & \\
\hline \multirow{2}{*}{$\mathrm{Ma}, 2019$ [19] } & 135 & \multirow{2}{*}{$\begin{array}{c}41 \\
(7-104.2)\end{array}$} & & $60.7 \%$ & $40.7 \%$ & M51.2 & $57.0 \%$ & $45.2 \%$ & \multirow{2}{*}{$\begin{array}{c}\text { MVA, OS: Stage } \\
\text { (<0.001); LNR } \\
<0.001 \text {; total vs. } \\
\text { subtotal gastrectomy } \\
0.007) ; \text { tumor } \\
\text { deposit }(0.028)\end{array}$} & $\begin{array}{c}\text { Overall LRR [ } n=135 \\
\text { (CRT) } 280 \text { (CTx)] } 7.4 \% \text { vs. }\end{array}$ & \multirow{2}{*}{$\begin{array}{c}\text { Leukopenia ( } 16.3 \% \text { vs. } 13.3 \% \\
p=0.493) \text {; anorexia }(14.8 \% \text { vs. } \\
11.1 \%, p=0.365) ; \mathrm{N} / \mathrm{V}(8.9 \% \text { vs. } \\
7.4 \%, p=0.615)\end{array}$} \\
\hline & 135 & & & $52.6 \%$ & $16.3 \%$ & M39.3 & $38.5 \%$ & $19.3 \%$ & & $\begin{array}{c}p<0.001 \text { ); Overall DM } \\
21.5 \% \text { vs. } 21.1 \% \text { (CRT vs. } \\
\text { CT, } p=0.924 \text { ) }\end{array}$ & \\
\hline
\end{tabular}

Abbreviations: CRT, chemoradiation; CT, chemotherapy; DFS, disease-free survival; OS, overall survival; LRR, locoregional recurrence rate; DM, distant metastases; N/V, nausea and vomiting; GI, gastrointestinal; NS, not significant; Cx, complication; CTx, chemotherapy; UVA, univariate analysis; MVA, multivariate analysis; LNR, lymph node ratio. Statistically significant $p$-values marked in bold font. 
The overall pooled OR for overall survival (OS) was 1.124 (95\% CI: 0.881-1.434, $p=0.347$ ). The pooled ORs of subgroup analyses of RCTs and the balanced studies were 1.208 (95\% CI: 0.921-1.584, $p=0.172$ ) and 1.279 (95\% CI: 0.995-1.644, $p=0.055$ ), respectively. A medium degree of heterogeneity was found in overall pooled analysis and subgroup analysis of the balanced studies, and the subgroup analysis of RCTs alone had very low heterogeneity. Considering the marginally significant result in the subgroup analysis of RCTs alone, in a further subgroup analysis of studies involving stage $\geq$ III patients only, the pooled result was statistically significant with an OR of 1.663 (95\% CI: 1.170-2.363, $p=0.005$ ). The pooled 3-year OS rates in the single-arm analyses were $61.4 \%$ (95\% CI: 51.1-70.8) and $59.1 \%$ (95\% CI: 44.4-72.3) in the CRT and CT arms, respectively; the corresponding 5-year OS rates were $55.8 \%$ (95\% CI: 44.5-66.5) and 46.9\% (95\% CI: 32.0-62.4), respectively. The heterogeneity among studies was very high and significant on single-arm analysis. Prognosticators of OS and DFS were available in four and two studies, respectively. Regarding OS prognosticators, cancer stage was significant in three of the four studies, and positive lymph nodes in surgical pathology or a high lymph node ratio (i.e., number of positive lymph nodes/total dissected lymph nodes) was significant in all four studies. Among DFS prognosticators, the lymph node ratio was significant in one study, and cancer stage and age were significant in the other study. Forest plots of the pooled analyses of 3-year DFS and OS rates are shown in Figure 2.

The LRR rates were significantly lower in the CRT arms than in the CR arms. The pooled OR was 0.559 (95\% CI: $0.355-0.879, p=0.012)$ for all studies, with a medium degree of heterogeneity $(p=0.073$, $\left.\mathrm{I}^{2}=46.1 \%\right)$. In subgroup analyses of RCTs, the OR was 0.495 (95\% CI: $\left.0.342-0.715, p<0.001\right)$ with very low heterogeneity $\left(p=0.569, \mathrm{I}^{2}=\sim 0.0 \%\right)$. In the balanced studies, the OR was 0.472 ( $95 \% \mathrm{CI}$ : $0.334-0.667)$ with a low degree of heterogeneity $\left(p=0.357, \mathrm{I}^{2}=9.2 \%\right)$. Single-arm analyses revealed overall pooled LRR rates of $11.3 \%$ (95\% CI: 7.5-1.68) and 18.1\% (95\% CI: 13.1-24.4) in the CRT and CR arms, respectively. As for the distant recurrence rate (DRR), the OR was 0.768 (95\% CI: 0.611-0.965, $p=0.023)$ favoring the CRT arm, with very low heterogeneity $\left(p=0.504, \mathrm{I}^{2}=\sim 0.0 \%\right)$. However, there was no statistically significant difference in the subgroup analysis of RCTs alone (OR: $0.788,95 \%$ CI: $0.589-1.054, p=0.108)$, whereas borderline significance was noted when analyzing balanced studies alone (OR: $0.802,95 \%$ CI: $0.634-1.015, p=0.066$ ). DRR rates on pooled single-arm analyses were $24.1 \%$ (95\% CI: 18.1-31.2) and 30.9\% (95\% CI: 24.4-38.3) in the CRT and CT arms, respectively. Heterogeneities were high and significant on single-arm pooled analyses of LRR and DRR (Figure 3A,B).

The most common grade $\geq 3$ complications reported were leukopenia and nausea and/or vomiting. Leukopenia was reportedly higher in the CRT arm than in the CR arm, with a pooled OR of 1.387 (95\% CI: 1.109-1.734, $p=0.004$ ). Nausea and/or vomiting was not significantly different between the two arms, with an OR of 1.224 (95\% CI: $0.893-1.677, p=0.209$ ). Heterogeneity was very low in both the abovementioned analyses. In single-arm pooled analyses, the incidence rates of leukopenia were $26.4 \%$ (95\% CI: 17.7-37.5) and 15.7\% (95\% CI: 9.4-25.0) in CRT and CT arms, respectively, and the incidence rates of nausea and/or vomiting were $11.2 \%$ (95\% CI: 7.8-15.8) and $11.1 \%$ (95\% CI: 7.6-16.0) in CRT and CT arms, respectively. Heterogeneity was high in both single-arm analyses (Figure 3C). Regarding grade 5 toxicity, one fatal complication was found in each arm of the pooled studies.

Possible publication bias was observed in the overall pooled analysis of DRR, the subgroup analysis of OS of studies involving stage $\geq$ III patients only, and single-arm analyses of leukopenia and nausea and/or vomiting. Sensitivity analyses using Duval and Tweedie's method revealed that, in the overall pooled analysis of DRR, the OR was mildly increased, and the statistical significance of the difference was decreased (trimmed OR: 0.837, 95\% CI: 0.655-1.070). Regarding the subgroup analysis of OS with studies involving stage $\geq$ III patients only, trimmed OR was increased to 1.963 , which reflects a more significant result. The results of all the pooled analyses are detailed in Table 3. 
(A)

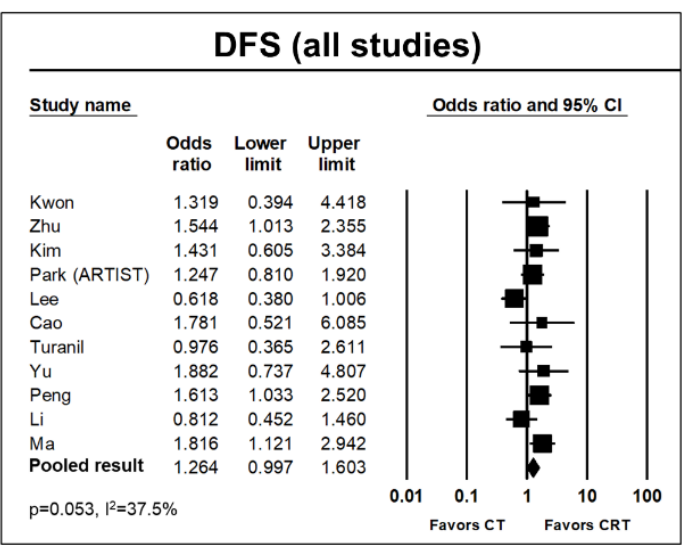

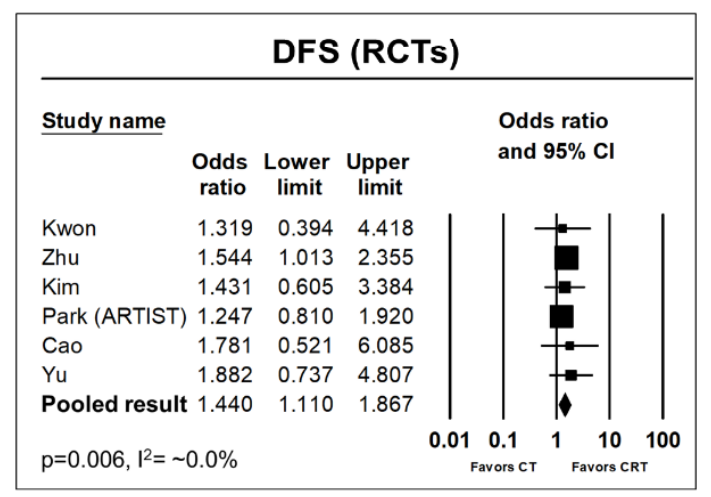

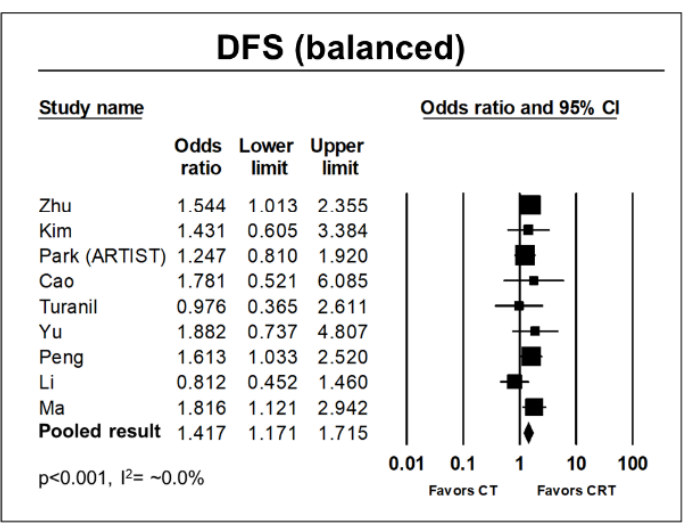

(B)

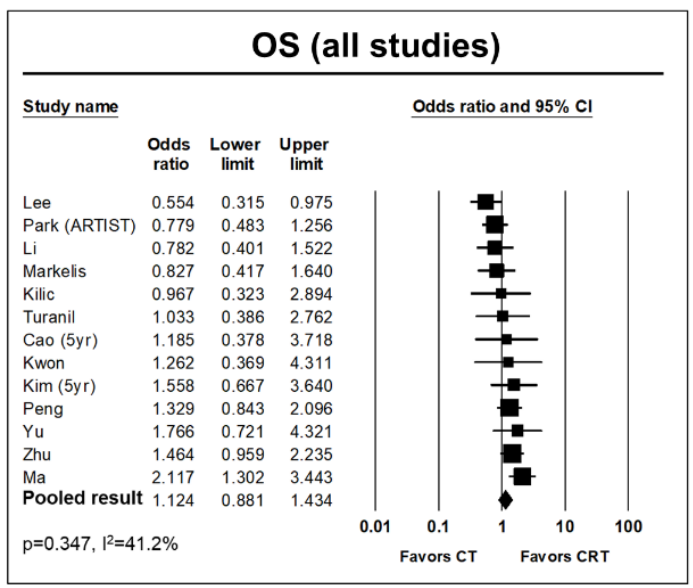

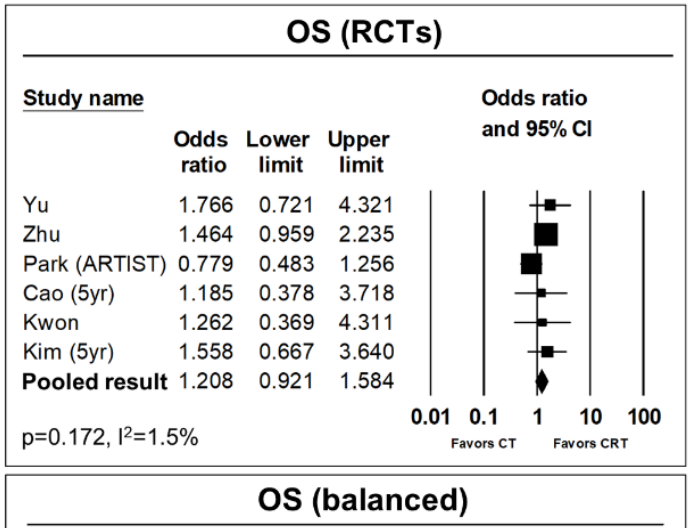

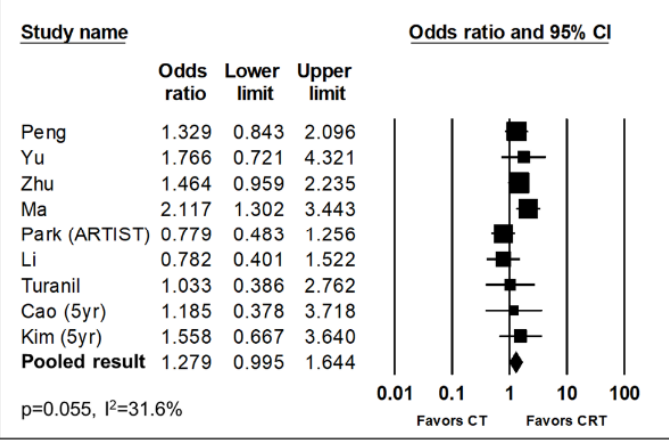

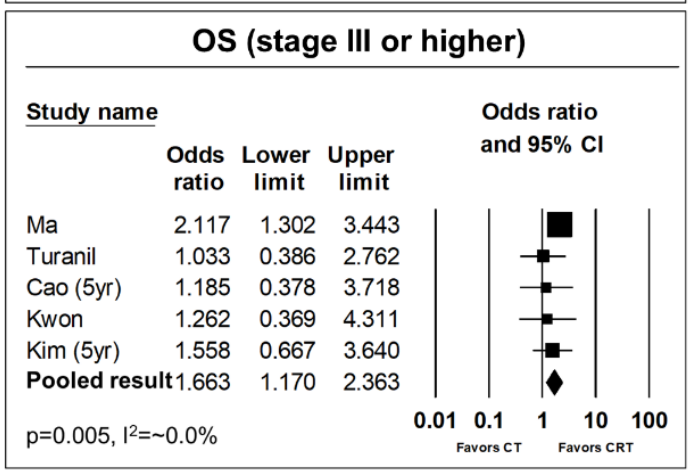

Figure 2. (A) Forest plots of overall and subgroup pooled analyses of 3-year disease-free survival (DFS). (B) Forest plots of overall and subgroup pooled analyses of 3-year overall survival (OS). CI, confidence interval; $\mathrm{RCT}$, randomized controlled trial. 
(A)
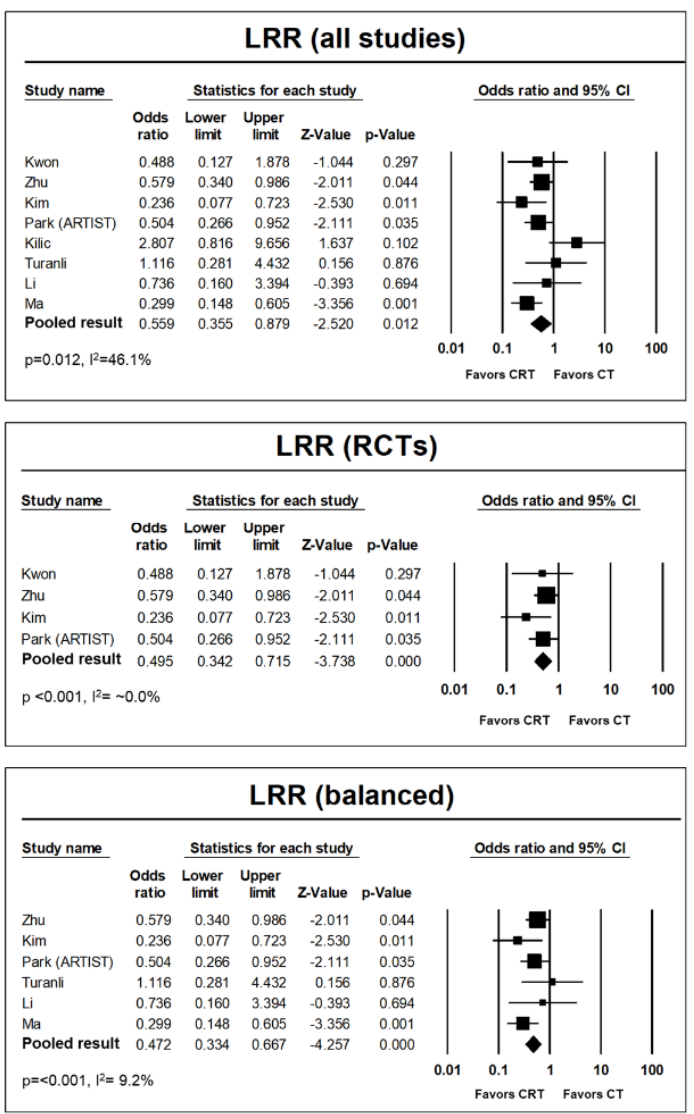

(B)
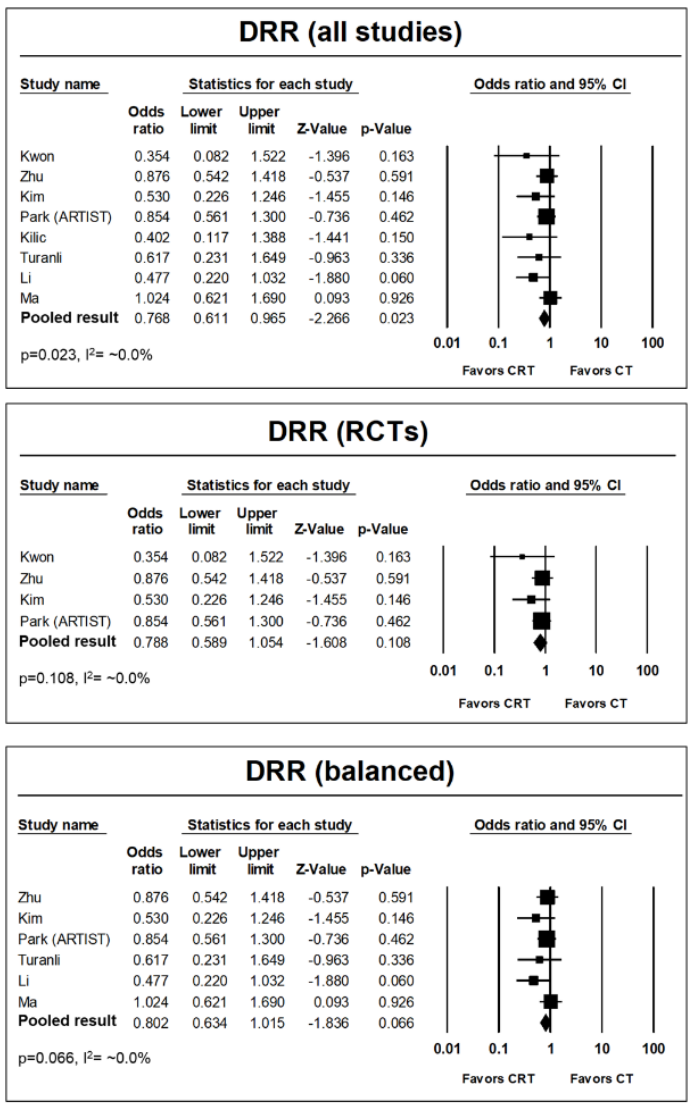

(C)

\begin{tabular}{|c|c|c|c|c|c|c|}
\hline \multicolumn{7}{|c|}{ Leukopenia grade 3 or higher } \\
\hline \multirow[t]{2}{*}{ Study name } & & & & \multicolumn{3}{|c|}{ Odds ratio and $95 \% \mathrm{Cl}$} \\
\hline & $\begin{array}{l}\text { Odds } \\
\text { ratio }\end{array}$ & $\begin{array}{c}\text { Lower } \\
\text { limit }\end{array}$ & $\begin{array}{c}\text { Upper } \\
\text { limit }\end{array}$ & & & \\
\hline Zhu & 1.030 & 0.462 & 2.294 & & & \\
\hline Kim & 0.731 & 0.270 & 1.984 & & & \\
\hline Park (ARTIST) & 1.367 & 0.944 & 1.978 & & & \\
\hline Cao & 1.962 & 0.621 & 6.193 & & & \\
\hline Yu & 1.332 & 0.278 & 6.378 & & & \\
\hline Peng & 1.268 & 0.795 & 2.024 & & & \\
\hline $\mathrm{Li}$ & 2.819 & 1.441 & 5.514 & & $\rightarrow-$ & \\
\hline \multirow[t]{3}{*}{ Ma } & 1.269 & 0.647 & 2.493 & & & \\
\hline & 1.387 & 1.109 & 1.734 & & $\downarrow$ & \\
\hline & & & & $0.01 \quad 0.1$ & 10 & 100 \\
\hline
\end{tabular}

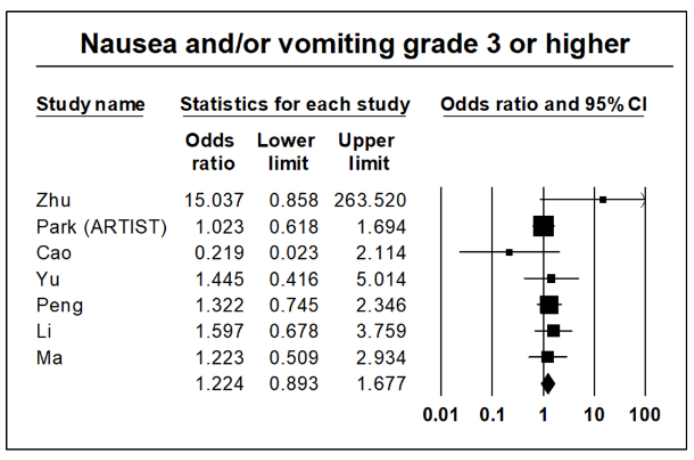

Figure 3. (A) Forest plots of overall and subgroup pooled analyses of locoregional recurrence rate (LRR). (B) Forest plots of overall and subgroup pooled analyses of distant recurrence rate (DRR). CI, confidence interval; RCT, randomized controlled trial. (C) Forest plots of pooled analyses of leukopenia and nausea and/or vomiting. 
Table 3. Pooled results of endpoints.

\begin{tabular}{|c|c|c|c|c|c|c|c|c|c|}
\hline Studies & No. of Studies & No. of Patients & Heterogeneity $p$ & $I^{2}(\%)$ & Heterogeneity & Pooled Results $(95 \%$ CI) & $p$ (Pooled Analyses) & Egger's $p$ & \\
\hline \multicolumn{9}{|c|}{ DFS, controlled comparisons } & \\
\hline All studies & 11 & 2379 & 0.1 & $37.5 \%$ & Medium & OR $1.264(0.997-1.603)$ & 0.053 & 0.942 & \\
\hline RCTs & 6 & 1089 & 0.964 & $\sim 0.0 \%$ & Very low & OR 1.440 (1.110-1.867) & 0.006 & 0.420 & \\
\hline Balanced & 9 & 1913 & 0.61 & $\sim 0.0 \%$ & Very low & OR 1.417 (1.171-1.715) & $<0.001$ & 0.824 & \\
\hline \multicolumn{9}{|c|}{ 3-year DFS, single-arm analysis } & \\
\hline CRT arm & 11 & 1225 & $<0.001$ & $84.9 \%$ & Very high & $62.5 \%(54.6-69.8)$ & NA & 0.749 & \\
\hline $\mathrm{CT}$ arm & 11 & 1154 & $<0.001$ & $91.3 \%$ & Very high & $57.1 \%(46.1-67.5)$ & NA & 0.868 & \\
\hline \multicolumn{9}{|c|}{ 5-year DFS, single-arm analysis } & \\
\hline CRT arm & 8 & 848 & $<0.001$ & $91.1 \%$ & Very high & $53.3 \%(40.9-65.4)$ & NA & 0.851 & \\
\hline $\mathrm{CT}$ arm & 8 & 861 & $<0.001$ & $93.4 \%$ & Very high & $40.9 \%(27.3-56.0)$ & NA & 0.748 & \\
\hline \multicolumn{9}{|c|}{ OS, controlled comparisons } & \\
\hline All studies & 13 & 2583 & 0.06 & $41.2 \%$ & Medium & OR 1.124 (0.881-1.434) & 0.347 & 0.760 & \\
\hline RCTs & 6 & 1089 & 0.406 & $1.5 \%$ & Very low & OR $1.208(0.921-1.584)$ & 0.172 & 0.622 & \\
\hline Balanced & 9 & 1913 & 0.166 & $31.6 \%$ & Medium & OR 1.279 (0.995-1.644) & 0.055 & 0.840 & trimmed value $^{\dagger}$ \\
\hline Stage $\geq$ III & 5 & 563 & 0.662 & $\sim 0.0 \%$ & Very low & OR $1.663(1.170-2.363)$ & 0.005 & 0.023 & $\begin{array}{c}\text { OR 1.963 } \\
(1.443-2.671)\end{array}$ \\
\hline \multicolumn{9}{|c|}{ 3-year OS, single-arm analysis } & \\
\hline CRT arm & 11 & 1235 & $<0.001$ & $90.8 \%$ & Very high & $61.4 \%(51.1-70.8)$ & NA & 0.479 & \\
\hline CT arm & 11 & 1208 & $<0.001$ & $95.0 \%$ & Very high & $59.1 \%(44.4-72.3)$ & NA & 0.513 & \\
\hline \multicolumn{9}{|c|}{ 5-year OS, single-arm analysis } & \\
\hline CRT arm & 8 & 848 & $<0.001$ & $89.1 \%$ & Very high & $55.8 \%(44.5-66.5)$ & NA & 0.885 & \\
\hline $\mathrm{CT}$ arm & 8 & 861 & $<0.001$ & $93.9 \%$ & Very high & $46.9 \%(32.0-62.4)$ & NA & 0.922 & \\
\hline \multicolumn{9}{|c|}{ Locoregional recurrence, controlled comparison } & \\
\hline All studies & 8 & 1724 & 0.073 & $46.1 \%$ & Medium & OR 0.559 (0.355-0.879) & 0.012 & 0.439 & \\
\hline RCTs & 4 & 960 & 0.569 & $\sim 0.0 \%$ & Very low & OR $0.495(0.342-0.715)$ & $<0.001$ & 0.307 & \\
\hline Balanced & 6 & 1592 & 0.357 & $9.2 \%$ & Low & OR $0.472(0.334-0.667)$ & $<0.001$ & 0.863 & \\
\hline
\end{tabular}


Table 3. Cont

\begin{tabular}{|c|c|c|c|c|c|c|c|c|c|}
\hline Studies & No. of Studies & No. of Patients & Heterogeneity $p$ & $\mathrm{I}^{2}(\%)$ & Heterogeneity & Pooled Results (95\% CI) & $p$ (Pooled Analyses) & Egger's $p$ & \\
\hline \multicolumn{9}{|c|}{ Locoregional recurrence, single-arm analysis } & \\
\hline CRT arm & 8 & 810 & 0.001 & $70.4 \%$ & High & $11.3 \%(7.5-16.8)$ & NA & 0.786 & \\
\hline $\mathrm{CT}$ arm & 8 & 914 & $<0.001$ & $73.7 \%$ & High & $18.1 \%(13.1-24.4)$ & NA & 0.459 & \\
\hline \multicolumn{10}{|c|}{ Distant metastasis, controlled comparison } \\
\hline All studies & 8 & 1724 & 0.504 & $\sim 0.0$ & Very low & OR $0.768(0.611-0.965)$ & 0.023 & 0.004 & $\begin{array}{c}\text { OR 0.837 } \\
(0.655-1.070)\end{array}$ \\
\hline RCTs & 4 & 960 & 0.511 & $\sim 0.0$ & Very low & OR 0.788 (0.589-1.054) & 0.108 & 0.027 & $\begin{array}{c}\text { OR } 0.814 \\
(0.612-1.083)\end{array}$ \\
\hline Balanced & 6 & 1592 & 0.543 & $\sim 0.0$ & Very low & OR $0.802(0.634-1.015)$ & 0.066 & 0.051 & $\begin{array}{c}\text { OR } 0.908 \\
(0.710-1.160)\end{array}$ \\
\hline \multicolumn{10}{|c|}{ Distant metastasis, single-arm analysis } \\
\hline CRT arm & 8 & 810 & $<0.001$ & $74.3 \%$ & High & $24.1 \%(18.1-31.2)$ & NA & 0.621 & \\
\hline CT arm & 8 & 914 & $<0.001$ & $75.3 \%$ & High & $30.9 \%(24.4-38.3)$ & NA & 0.069 & $29.0 \%(22.6-36.3)$ \\
\hline \multicolumn{10}{|c|}{ Complication of grade $\geq 3$} \\
\hline Leukopenia & 8 & 1821 & 0.433 & $\sim 0.0$ & Very low & OR 1.387 (1.109-1.734) & 0.004 & 0.946 & \\
\hline $\mathrm{N} / \mathrm{V}$ & 7 & 1731 & 0.406 & $2.5 \%$ & Very low & OR $1.224(0.893-1.677)$ & 0.209 & 0.617 & \\
\hline \multicolumn{10}{|c|}{ Leukopenia, single-arm analysis } \\
\hline CRT arm & 10 & 1154 & $<0.001$ & $91.9 \%$ & Very high & $26.4 \%(17.7-37.5)$ & NA & 0.105 & \\
\hline $\mathrm{CT}$ arm & 10 & 1133 & $<0.001$ & $91.1 \%$ & Very high & $15.7 \%(9.4-25.0)$ & NA & 0.074 & $17.1 \%(10.7-26.2)$ \\
\hline \multicolumn{10}{|c|}{$\mathrm{N} / \mathrm{V}$, single-arm analysis } \\
\hline CRT arm & 9 & 1108 & $<0.001$ & $72.6 \%$ & High & $11.2 \%(7.8-15.8)$ & NA & 0.200 & \\
\hline $\mathrm{CT}$ arm & 9 & 1089 & 0.005 & $63 . .8 \%$ & High & $11.1 \%(7.6-16.0)$ & NA & 0.014 & $11.7 \%(7.7-17.5)$ \\
\hline
\end{tabular}

Abbreviations: CI, confidence interval; OR, odds ratio; RCT, randomized controlled trial; CRT, chemoradiation; CT, chemotherapy; NA, not assessable; DFS, disease-free survival; OS, overall survival; N/V, nausea and/or vomiting. ${ }^{\dagger}$ Values from Duval and Tweedie's trim and fill method. 


\section{Discussion}

\subsection{Clinical Interpretation of Pooled Results and Related Literature}

Gastric cancer has a tendency to spread locoregionally and hematogenously, even after an extended surgical approach [16]. The addition of radiotherapy to neoadjuvant or adjuvant CT could further reduce LRR by eradicating subclinical tumor foci in the perigastric region. CRT has been applied in both neoadjuvant and adjuvant settings. The German POET (Preoperative chemotherapy versus chemoradiotherapy in locally advanced adenocarcinomas of the oesophagogastric junction) trial reported a higher pathologic complete remission and node-negative status and extended OS (3-year: $47.4 \%$ vs. $27.7 \%$ ) compared with neoadjuvant CT alone [33]. Another RCT from Nordic countries reported results with similar trends, with higher pathologic complete remission, lymph node negative status, and R0 status [34]. Although these results were significant, most of the relevant studies were conducted on patients who were diagnosed with diseases in the esophagus or gastroesophageal junction. Therefore, extrapolating these results to other populations, specifically Asians, who are generally diagnosed with stomach cancer that is mainly observed in the body or non-cardia area, might be irrelevant [3,35]. Considering its high incidence in Asia and southern Europe, several clinical guidelines recommend upfront D2 gastrectomy for cases, except in very early disease (e.g., T1N0), and discuss the necessity of adjuvant treatment including CT and CRT [2-4,15].

Our study's first take-home message is that the application of CRT after D2 resection has a clear oncologic benefit in terms of DFS and LRR. The more significant results in the subgroup analyses with markedly lower heterogeneity reflect a clearer oncologic benefit with better clinical distribution between the two arms. Considering the difference in DFS rates between the CRT and CT arms, which was wider at 5 years (53.3\% vs. $40.9 \%)$ than at 3 years (62.5\% vs. $57.1 \%)$ on single-arm analysis, mid- to long-term locoregional control with CRT may have contributed to a longer DFS. In a previous surgical series, locoregional and hematogenous recurrences occurred in 32.5\% and 34.3\% of 1500 patients, respectively, who underwent D2 gastrectomy and experienced subsequent failure; their corresponding mean survival periods were similar (11.0 and 9.4 months, respectively) [16]. Patients in that study who underwent curative resection for LRR had a mean survival of 21.6 months. These results demonstrate that locoregional and hematogenous recurrences markedly impact oncologic outcomes, indicating that longer survival might be achieved by reducing LRR. Furthermore, Chang et al. [36]. reported that the most prevalent nodal recurrences among patients with stage III gastric cancer were outside the D2 dissection field; hence, adjuvant radiotherapy might control recurrences in those nodal basins.

Although DRR decreased according to the overall pooled analysis, these findings were not significant in the subgroup analysis with RCTs alone. Cancers tend to occur step-wise, developing locally in the primary site and ultimately spreading systemically [37]. Controlling LRR might affect DRR according to previous studies of other malignancies [38,39], but data regarding gastric cancer are insufficient. The possible correlation between LRR reduction via CRT and improved DRR should be further investigated.

Although DFS was shown to be an acceptable surrogate for OS in gastric cancer [40], improvements in DFS and LRR might be insufficient to significantly alter common practice and overcome concerns regarding additional toxicities. The next step for optimizing CRT for gastric cancer is to identify patients who can most benefit from LRR reduction and ultimately experience longer OS. Peng et al. [31] reported improved OS with CRT compared with CT in their subgroup of patients with stage IIIC disease (median OS: 29 vs. 23 months, $p=0.049$ ). A study by Ma et al. [19], which was one of the largest series performed, revealed that CRT provided an OS benefit over CT among patients with stage IIIA and IIIB disease (the 5-year OS rates for stage IIIA patients were $61.5 \%$ vs. 34.5\% $p=0.03$; those for IIIB patients were $46.4 \%$ vs. $26.1 \%, p=0.035)$. Although the result was not significant in overall pooled analysis, the subgroup analysis of studies involving stage $\geq$ III patients only showed a significant benefit regarding OS (OR 1.663, $p=0.005$ ), suggesting selective application of CRT after D2 gastrectomy for patients with locally advanced diseases. 
Regarding complications, the most problematic complication in response to radiation added to CT was leukopenia. Since hematopoietic cells are highly fragile to radiation exposure, reducing bystander irradiation with the application of intensity-modulated radiotherapy (IMRT) can reduce this complication [41,42]. Specific bone marrow-sparing technique can also be helpful in reducing leukopenia [43]. Of note, the study by Zhu et al. [18] has reported a significantly low rate of leukopenia $(7.5 \%)$ after CRT using IMRT and has shown a favorable survival in the CRT arm (median OS: 54 vs. 38 months, $p=0.122$ ). Furthermore, CRT should be carefully performed for patients with compromised immunity, such as those having malignancy related with common variable immunodeficiency $[44,45]$.

A limitation inherent to literature analysis is that the majority of studies are published in only a few countries in East Asia. Considering that gastric cancer is the third leading cause of cancer-related death globally, the literature remains significantly insufficient. Hence, with respect to administering CRT after D2 gastrectomy, major clinical guidelines depend almost entirely on data from the ARTIST trial [3,14,15], as guidelines from Japan [2] and Italy [4] do not even mention radiotherapy and CRT as possible modalities after D2 gastrectomy. Unfortunately, the ARTIST trial marginally failed to achieve the primary DFS endpoint $(p=0.0862)$ [12]; this result led to highly conservative recommendations of CRT after D2 resection in the guidelines, such as only for patients with remnant disease (R1 or R2 section) or resection with less-extensive lymphadenectomy (D0 or D1). However, as stated by the American Statistical Association, binary decisions solely depend on a predefined $p$-value that might cause serious misinterpretation [46]. Furthermore, the clinical significance of subgroup analyses and those of the entire targeted population should also be emphasized. In the ARTIST trial, subgroup analysis of $\mathrm{N}+$ patients showed a significant DFS benefit ( $p=0.0365)$, and nearly $90 \%$ of the patients belonged to various $\mathrm{N}+$ subgroups. When considering this, the ARTIST trial may not have actually "failed" as deemed in several clinical guidelines [2-4,14,15]. Moreover, although complications tended to increase in the CRT arms of the present meta-analysis, most were transient, and grade 5 complications were significantly rare. Wider application of modern radiotherapy techniques is expected to diminish toxicities, as mentioned above [41-43]. Taken altogether, the application of CRT after D2 gastrectomy should be considered for patients with high-risk of LRR, and future randomized studies should identify specific subgroups that can derive OS benefit from CRT, prompted by locoregional benefits and the application of modern techniques.

\subsection{Practical Implication and Future Perspectives}

Adjuvant CRT after D2 gastrectomy is not commonly practiced in clinical settings, despite its benefits in locoregional control [3]. Although our results cannot change current clinical practice in a short time, our study might at least stimulate the conduction of clinical trials applying adjuvant CRT, specifically for advanced cases with stage $\geq$ III. Multidisciplinary approach has been more rarely performed for gastric cancer in clinical settings compared with other cancers. However, it was recently reported that the diagnoses of gastric cancer were changed in $18-27 \%$ of patients, and $23-77 \%$ of treatment policies were changed after multidisciplinary discussion [47-49]. The results of our study will further increase the necessity of multidisciplinary discussions to optimize clinical decision.

Several molecular pathways are known to be involved in gastric carcinogenesis, such as human epidermal grow th factor receptor 2 (HER2), HER3, epidermal growth factor receptor (EGFR), hepatocyte growth factor receptor/c-MET, E-cadherin, matrix metalloproteinase, vascular endothelial growth factor (VEGF)/VEGF receptor (VEGFR), WNT/ $\beta$-catenin, fibroblast growth factor receptor, and $\mathrm{Akt} / \mathrm{PI} 3 \mathrm{~K} / \mathrm{mTOR}$, in gastric cancer and other gastrointestinal (GI) malignancies [50]. Additional administration of trastuzumab, a recombinant monoclonal antibody against HER2, has shown a better significant survival benefit compared with conventional CT alone [51]. A monoclonal antibody targeting VEGFR-2, ramucirumab, has also shown significant benefit as a second-line systemic treatment agent [52,53]. Novel molecular findings regarding drug resistance and regional dissemination of GI cancers will stimulate the development of future systemic agents to overcome these clinical hindrances [54,55]. So far, CRT has been only studied as a radiotherapy combined with conventional 
$\mathrm{CT}$, either in a neoadjuvant or adjuvant setting $[3,13]$. The results of the present study will encourage future studies optimizing gastric cancer treatment by combining radiotherapy with target agents or newer agents actively being researched.

\subsection{Limitations}

A limitation of this meta-analysis was that the overall numbers of studies and of RCTs with a sufficient number of enrolled patients were small. A meta-analysis increases the sample size and, consequently, the power to study the effects of interest by combining primary studies while considering the sizes of the studies included. However, one number cannot summarize a research field and can be affected by the heterogeneity of studies, by publication bias and by the fact that not all variables are comparable despite the performance of complementing statistical methods. Clinical application of meta-analysis results should be based on the interpretation of both pooled data and detailed information from individual studies that reflect actual clinical practices [56]. Meta-analyses of non-randomized controlled studies (NRCTs) have been controversial because their potential heterogeneity might skew the pooled analyses; however, oncology research cannot always derive the firmest evidence from RCTs, and carefully performed meta-analyses including NRCTs might provide clinically useful information in obscure areas [57]. Previous systematic reviews also found that meta-analyses based on RCTs versus high-quality studies had similar outcome estimates [58]. Compared with previous meta-analyses of related subjects [59-61], our study included the largest number of RCTs, and patients were analyzed from real-world data and NRCTs with no language restrictions during searching. Furthermore, by performing sensitivity analyses (including RCTs and balanced studies), we attempted to minimize the potential bias of including NRCTs and to improve the reliability of outcomes involving real-world data.

\section{Methods}

This study was designed to answer the PICO question "is there an oncologic benefit of adjuvant CRT compared to CT after D2 resection for gastric cancer in a real-world clinical setting?" and adhered to the Preferred Reporting Items for Systematic Reviews and Meta-Analyses guidelines. A systematic search of the PubMed, MEDLINE, Embase, and Cochrane Library databases was performed up to 20 January 2020, using the following search terms: "(gastric OR stomach) AND cancer AND (radiotherapy OR 'radiation therapy') AND survival AND D2." No language or publishing period restriction was applied. Full-text publications were searched to identify a rational clinical comparison between the CRT and CT arms.

\subsection{Inclusion Process and Criteria}

The primary endpoint of the study was DFS and OS. The secondary endpoints included LRR, DRR, and grade $\geq 3$ complication rates. Our inclusion criteria were as follows: (1) controlled clinical trials aimed at comparing CRT and CT after D2 resection for gastric cancer, (2) inclusion of at least 10 patients in each arm, and (3) reporting of at least one survival outcome (DFS or OS).

After the initial search, duplicate studies, conference abstracts, reviews, letters, editorials, case reports, laboratory studies, and irrelevant studies were filtered out using titles and citations. The remaining studies were assessed by reviewing their abstracts and full texts to identify those that met all of the inclusion criteria. We included multiple studies from the same institutions only if they did not have overlapping populations or if any overlap was negligible. For studies with possibly overlapping populations, we selected only one using the following criteria (prioritized in numerical order): (1) the study with a higher level of evidence (e.g., RCTs were preferred over NRCTs), (2) that with the larger number of patients, and (3) the most recently published. All studies were selected by two independent researchers who resolved any disagreements by mutual discussion. 


\subsection{Data Collection and Quality Assessment}

Data acquisition was performed by two independent researchers using a pre-standardized form, including background information of authors, affiliations, study design, patients recruiting periods, and number of patients; clinical information including treatment modality, patients' age, rate of diffuse type cancer cases, and T and N stages; clinical outcomes including DFS, OS, prognosticators of survival outcomes, pattern of failure, and grade 3 or higher toxicities. Missing DFS and OS rates were estimated from the descriptive graphs in consideration of the follow-up periods. For quality assessment of the included NRCTs and RCTs, the Newcastle-Ottawa scale was used [62]. Studies with scores of $\geq 8$ were considered high quality, scores of 7-8 medium quality, and the remainder low quality.

\subsection{Statistical Analyses}

Considering the range of clinical diversity, the different institutions with distinct treatment modalities, and the inclusion of studies (NRCTs and RCTs) of different designs, a random effects model was used for the pooled analysis of endpoints [63]. We performed pooled analyses in a stepwise-hierarchical manner; ORs calculated from the comparison of endpoints between the CRT and CT arms were pooled and analyzed for all studies, RCTs alone, and balanced studies alone. Balanced studies were defined as those without significant differences $(p<0.05)$ in the patients' clinical profiles (i.e., age, histologic type, and TNM stage). Heterogeneity among studies was evaluated using $\mathrm{I}^{2}$ statistics [64] and the Cochran $\mathrm{Q}$ test [65]. Significant heterogeneity was considered present when $p<0.1$ and $\mathrm{I}^{2} \geq 50 \% ; \mathrm{I}^{2}$ values of $25 \%, 50 \%$, and $75 \%$ corresponded to low, moderate, and high degrees of heterogeneity, respectively [66]. Visual inspection of funnel plots and the Egger's test [67] were used to identify possible publication biases. For pooled analyses with significant asymmetry in funnel plots or two-tailed $p$-values $<0.1$ in the Egger's test, Duval and Tweedie's trim and fill method [68] was performed for sensitivity analysis. All statistical analyses were designed and confirmed by both a clinician and a biostatistician specialized in meta-analysis and performed using Comprehensive Meta-Analysis version 3 (Biostat Inc., Englewood, NJ, USA).

\subsection{Ethical Consideration}

Ethical approval was not required because this study retrieved and synthesized data from already published studies. Otherwise, authors declare that the investigations were performed following the principles of the Declaration of Helsinki of 1975 [69], revised in 2013.

\section{Conclusions}

Our study clearly demonstrated the benefits of CRT after D2 gastrectomy in terms of DFS and LRR and also a possibility of decreased DRR. Although the result was not significant in overall pooled analysis, OS benefit was shown in the subgroup analysis of studies involving stage $\geq$ III patients only. Therefore, CRT after D2 gastrectomy should be applied for selected patients with a high risk of LRR, such as those with stage $\geq$ III disease, along with technical efforts such as IMRT or bone marrow-sparing technique, to reduce complications including leukopenia. Future randomized studies should focus on identifying specific subgroups of patients who can benefit from CRT after D2 gastrectomy considering OS.

Supplementary Materials: The following are available online at http://www.mdpi.com/2072-6694/12/8/2125/s1, Table S1: Study quality evaluation sheet.

Author Contributions: Conceptualization, writing—original draft preparation, writing—review and editing, data curation, C.H.R.; software, methodology, formal analysis, I.-S.S.; data curation, supervision, H.Y.L.; data curation, supervision, W.S.Y.; data curation, supervision, S.P. All authors have read and agreed to the published version of the manuscript.

Funding: This study was supported by the National Research Fund of Korea (NRF-2019M2D2A1A01031560). The research grant supported only methodological aspects including statistical analysis and linguistic correction and did not affect major contents including the results and conclusions. 
Acknowledgments: The authors appreciate the dedicated contribution of Hu Jing Hua, MSc., who majored in Korean Language Translation at Chonnam National University, Jeollanamdo, Korea, for his Chinese language translation.

Conflicts of Interest: The authors declare no conflict of interest.

\section{References}

1. Bray, F.; Ferlay, J.; Soerjomataram, I.; Siegel, R.L.; Torre, L.A.; Jemal, A. Global cancer statistics 2018: GLOBOCAN estimates of incidence and mortality worldwide for 36 cancers in 185 countries. CA Cancer J. Clin. 2018, 68, 394-424. [CrossRef] [PubMed]

2. Association, J.G.C. Japanese gastric cancer treatment guidelines 2014 (ver. 4). Gastric. Cancer 2017, 20 , 1-19. [CrossRef] [PubMed]

3. Guideline Committee of the Korean Gastric Cancer Association (KGCA), Development Working Group \& Review Panel. Korean Practice Guideline for Gastric Cancer 2018: An evidence-based, multi-disciplinary approach. J. Gastric Cancer 2019, 19, 1-48. [CrossRef]

4. De Manzoni, G.; Marrelli, D.; Baiocchi, G.L.; Morgagni, P.; Saragoni, L.; Degiuli, M.; Donini, A.; Fumagalli, U.; Mazzei, M.A.; Pacelli, F. The Italian Research Group for Gastric Cancer (GIRCG) guidelines for gastric cancer staging and treatment: 2015. Gastric Cancer 2017, 20, 20-30. [CrossRef]

5. Allum, W.; Meyer, H.; Garofalo, A.; Schuhmacher, J.; Demanzoni, G.; Degiuli, M.; Kulig, J.; van de Velde, C.; Roukos, D.; Barr, H. Gastric cancer in Europe: European union network of excellence (EUNE) for gastric cancer steering group. Br. J. Surg. 2008, 95, 406-408.

6. Degiuli, M.; Sasako, M.; Ponti, A.; Vendrame, A.; Tomatis, M.; Mazza, C.; Borasi, A.; Capussotti, L.; Fronda, G.; Morino, M. Randomized clinical trial comparing survival after D1 or D2 gastrectomy for gastric cancer. Br. J. Surg. 2014, 101, 23-31. [CrossRef]

7. Cunningham, D.; Allum, W.H.; Stenning, S.P.; Thompson, J.N.; Van de Velde, C.J.; Nicolson, M.; Scarffe, J.H.; Lofts, F.J.; Falk, S.J.; Iveson, T.J.; et al. Perioperative chemotherapy versus surgery alone for resectable gastroesophageal cancer. N. Engl. J. Med. 2006, 355, 11-20. [CrossRef]

8. Jansen, E.; Boot, H.; Dubbelman, R.; Verheij, M.; Cats, A. Postoperative chemoradiotherapy in gastric cancer-A phase I-II study of radiotherapy with dose escalation of weekly cisplatin and daily capecitabine chemotherapy. Ann. Oncol. 2010, 21, 530-534. [CrossRef]

9. Sasako, M.; Sakuramoto, S.; Katai, H.; Kinoshita, T.; Furukawa, H.; Yamaguchi, T.; Nashimoto, A.; Fujii, M.; Nakajima, T.; Ohashi, Y. Five-year outcomes of a randomized phase III trial comparing adjuvant chemotherapy with S-1 versus surgery alone in stage II or III gastric cancer. J. Clin. Oncol. 2011, 29, 4387-4393. [CrossRef]

10. Noh, S.H.; Park, S.R.; Yang, H.K.; Chung, H.C.; Chung, I.J.; Kim, S.W.; Kim, H.H.; Choi, J.H.; Kim, H.K.; $\mathrm{Yu}, \mathrm{W}$. Adjuvant capecitabine plus oxaliplatin for gastric cancer after D2 gastrectomy (CLASSIC): 5-year follow-up of an open-label, randomised phase 3 trial. Lancet Oncol. 2014, 15, 1389-1396. [CrossRef]

11. Macdonald, J.S.; Smalley, S.R.; Benedetti, J.; Hundahl, S.A.; Estes, N.C.; Stemmermann, G.N.; Haller, D.G.; Ajani, J.A.; Gunderson, L.L.; Jessup, J.M.; et al. Chemoradiotherapy after surgery compared with surgery alone for adenocarcinoma of the stomach or gastroesophageal junction. N. Engl. J. Med. 2001, 345, 725-730. [CrossRef] [PubMed]

12. Lee, J.; Lim, D.H.; Kim, S.; Park, S.H.; Park, J.O.; Park, Y.S.; Lim, H.Y.; Choi, M.G.; Sohn, T.S.; Noh, J.H.; et al. Phase III trial comparing capecitabine plus cisplatin versus capecitabine plus cisplatin with concurrent capecitabine radiotherapy in completely resected gastric cancer with D2 lymph node dissection: The ARTIST trial. J. Clin. Oncol. 2012, 30, 268-273. [CrossRef] [PubMed]

13. Smyth, E.; Verheij, M.; Allum, W.; Cunningham, D.; Cervantes, A.; Arnold, D. Gastric cancer: ESMO Clinical Practice Guidelines for diagnosis, treatment and follow-up. Ann. Oncol. 2016, 27, v38-v49. [CrossRef] [PubMed]

14. National Cancer Comprehensive Network. NCCN Guidelines Version 4.2019 Gastric Cancer. Available online: https://www.nccn.org/professionals/physician_gls/pdf/gastric.pdf (accessed on 30 January 2020).

15. Wang, F.H.; Shen, L.; Li, J.; Zhou, Z.W.; Liang, H.; Zhang, X.T.; Tang, L.; Xin, Y.; Jin, J.; Zhang, Y.J. The Chinese Society of Clinical Oncology (CSCO): Clinical guidelines for the diagnosis and treatment of gastric cancer. Cancer Commun. 2019, 39, 10. [CrossRef] 
16. Yoo, C.; Noh, S.H.; Shin, D.; Choi, S.; Min, J. Recurrence following curative resection for gastric carcinoma. Br. J. Surg. 2000, 87, 236-242. [CrossRef]

17. Park, S.H.; Sohn, T.S.; Lee, J.; Lim, D.H.; Hong, M.E.; Kim, K.-M.; Sohn, I.; Jung, S.H.; Choi, M.G.; Lee, J.H. Phase III trial to compare adjuvant chemotherapy with capecitabine and cisplatin versus concurrent chemoradiotherapy in gastric cancer: Final report of the adjuvant chemoradiotherapy in stomach tumors trial, including survival and subset analyses. J. Clin. Oncol. 2015, 33, 3130-3136. [CrossRef]

18. Zhu, W.G.; Xua, D.F.; Pu, J.; Zong, C.D.; Li, T.; Tao, G.Z.; Ji, F.Z.; Zhou, X.L.; Han, J.H.; Wang, C.S. A randomized, controlled, multicenter study comparing intensity-modulated radiotherapy plus concurrent chemotherapy with chemotherapy alone in gastric cancer patients with D2 resection. Radiother. Oncol. 2012, 104, 361-366. [CrossRef]

19. Ma, G.F.; Zhang, H.G.; Liu, J.; Chen, Y.X.; Xiao, H.; Wang, X.F.; He, J.; Zeng, Z.C.; Sun, J.; Liu, T.S. Benefit of adjuvant chemoradiotherapy in patients with pathological stage III gastric cancer. Cancer Manag. Res. 2019, 11, 6029. [CrossRef]

20. Turanli, S.; Atalay, C.; Berberoglu, U.; Gulben, K. Adjuvant chemoradiation versus chemotherapy for stage III gastric cancer after surgery with curative intent. J. Cancer Res. Ther. 2015, 11, 369. [CrossRef]

21. Kim, T.H.; Park, S.R.; Ryu, K.W.; Kim, Y.-W.; Bae, J.-M.; Lee, J.H.; Choi, I.J.; Kim, Y.-J.; Kim, D.Y. Phase 3 trial of postoperative chemotherapy alone versus chemoradiation therapy in stage III-IV gastric cancer treated with R0 gastrectomy and D2 lymph node dissection. Int. J. Radiat. Biol. Phys. 2012, 84, e585-e592. [CrossRef]

22. Trip, A.K.; Nijkamp, J.; van Tinteren, H.; Cats, A.; Boot, H.; Jansen, E.P.M.; Verheij, M. IMRT limits nephrotoxicity after chemoradiotherapy for gastric cancer. Radiother Oncol. 2015, 114, 421-426. [CrossRef]

23. Boda-Heggemann, J.; Weiss, C.; Schneider, V.; Hofheinz, R.-D.; Haneder, S.; Michaely, H.; Wertz, H.; Ronellenfitsch, U.; Hochhaus, A.; Wenz, F. Adjuvant IMRT/XELOX radiochemotherapy improves long-term overall-and disease-free survival in advanced gastric cancer. Strahlenther. Onkol. 2013, 189, 417-423. [CrossRef] [PubMed]

24. Ringash, J.; Perkins, G.; Brierley, J.; Lockwood, G.; Islam, M.; Catton, P.; Cummings, B.; Kim, J.; Wong, R.; Dawson, L. IMRT for adjuvant radiation in gastric cancer: A preferred plan? Int. J. Radiat. Biol. Phys. 2005, 63, 732-738. [CrossRef] [PubMed]

25. Markelis, R.; Endzinas, Ž.; Kiudelis, M.; Grižas, S.; Pundzius, J.; Saladžinskas, Ž.; Juozaitytè, E.; Inčiūra, A.; Pranys, D.; Maleckas, A. Adjuvant therapy after curative resection with D2 lymphadenectomy for gastric cancer: Results of a prospective clinical trial. Medicina 2009, 45, 460. [CrossRef] [PubMed]

26. Kwon, H.C.; Kim, M.C.; Kim, K.H.; Jang, J.S.; Oh, S.Y.; KIM, S.H.; Kwon, K.A.; Lee, S.; Lee, H.S.; KIM, H.J. Adjuvant chemoradiation versus chemotherapy in completely resected advanced gastric cancer with D2 nodal dissection. Asia Pac. J. Clin. Oncol. 2010, 6, 278-285. [CrossRef]

27. Kilic, L.; Ordu, C.; Ekenel, M.; Yildiz, I.; Keskin, S.; Sen, F.; Gural, Z.; Asoglu, O.; Kizir, A.; Aykan, F. Comparison of two different adjuvant treatment modalities for $\mathrm{pN} 3$ gastric cancer patients after D2 lymph node dissection: Can we avoid radiotherapy in a subgroup of patients? Med. Oncol. 2013, 30, 660. [CrossRef]

28. Lee, S.J.; Sohn, T.S.; Lee, J.; Park, S.H.; Park, J.O.; Do Hoon, L.; Park, Y.S.; Lim, H.Y.; Choi, M.G.; Lee, J.H. Adjuvant chemoradiation with 5-fluorouracil/leucovorin versus S-1 in gastric cancer patients following D2 lymph node dissection surgery: A feasibility study. Anticancer Res. 2014, 34, 6585-6591.

29. Cao, X.G. Efficacy and adverse reactions of chemoradiotherapy after D2 radical resection for advanced gastric cancer. J. Pract. Oncol. 2015, 30, 533-536.

30. Yu, J.W.; Hao, J.Q.; Hu, Z.G.; Qian, L.T. Chemoradiotherapy vs pure chemotherapy for local progressive gastric cancer following D2 lymph node dissection: A comparison of effectiveness. Acad. J. Second Mil. Med. Univ. 2016, 37, 177-183.

31. Peng, J.; Wei, Y.; Zhou, F.; Dai, J.; Zhong, Y.; Xie, C.; Qin, Y.; Gong, J.; Xiong, B.; Zhou, Y. D2-resected stage IIIc gastric cancer patients benefit from adjuvant chemoradiotherapy. Cancer Med. 2016, 5, 2773-2780. [CrossRef]

32. Li, Q.; Li, G.; Palmer, J.D.; Zhang, Z. Lymph node burden as a predictive factor for selective chemoradiotherapy in patients with locally advanced gastric cancer after a D2 dissection. Amer. J. Clin. Oncol. 2017, 40, 375-380. [CrossRef] [PubMed]

33. Stahl, M.; Walz, M.K.; Riera-Knorrenschild, J.; Stuschke, M.; Sandermann, A.; Bitzer, M.; Wilke, H.; Budach, W. Preoperative chemotherapy versus chemoradiotherapy in locally advanced adenocarcinomas of the oesophagogastric junction (POET): Long-term results of a controlled randomised trial. Eur. J. Cancer (Oxford, England: 1990) 2017, 81, 183-190. [CrossRef] 
34. Klevebro, F.; Alexandersson von Döbeln, G.; Wang, N.; Johnsen, G.; Jacobsen, A.B.; Friesland, S.; Hatlevoll, I.; Glenjen, N.I.; Lind, P.; Tsai, J.A.; et al. A randomized clinical trial of neoadjuvant chemotherapy versus neoadjuvant chemoradiotherapy for cancer of the oesophagus or gastro-oesophageal junction. Ann. Oncol. 2016, 27, 660-667. [CrossRef] [PubMed]

35. Shim, J.H.; Song, K.Y.; Jeon, H.M.; Park, C.H.; Jacks, L.M.; Gonen, M.; Shah, M.A.; Brennan, M.F.; Coit, D.G.; Strong, V.E. Is gastric cancer different in Korea and the United States? Impact of tumor location on prognosis. Ann. Surg. Oncol. 2014, 21, 2332-2339. [CrossRef] [PubMed]

36. Chang, J.S.; Lim, J.S.; Noh, S.H.; Hyung, W.J.; An, J.Y.; Lee, Y.C.; Rha, S.Y.; Lee, C.G.; Koom, W.S. Patterns of regional recurrence after curative D2 resection for stage III (N3) gastric cancer: Implications for postoperative radiotherapy. Radiother Oncol. 2012, 104, 367-373. [CrossRef]

37. Brady, L.W.; Perez, C.A.; Wazer, D.E. Perez E Brady's Principles and Practice of Radiation Oncology; Lippincott Williams \& Wilkins: Philadelphia, PA, USA, 2013.

38. Kwong, D.; Sham, J.; Choy, D. The effect of loco-regional control on distant metastatic dissemination in carcinoma of the nasopharynx: An analysis of 1301 patients. Int. J. Radiat. Biol. Phys. 1994, 30, 1029-1036. [CrossRef]

39. Vicini, F.A.; Kestin, L.; Huang, R.; Martinez, A. Does local recurrence affect the rate of distant metastases and survival in patients with early-stage breast carcinoma treated with breast-conserving therapy? Cancer 2003, 97, 910-919. [CrossRef]

40. Oba, K.; Paoletti, X.; Alberts, S.; Bang, Y.J.; Benedetti, J.; Bleiberg, H.; Catalano, P.; Lordick, F.; Michiels, S.; Morita, S. Disease-free survival as a surrogate for overall survival in adjuvant trials of gastric cancer: A meta-analysis. J. Natl. Cancer Inst. 2013, 105, 1600-1607. [CrossRef]

41. Wang, X.; Zhao, D.; Yang, L.; Chi, Y.; Tang, Y.; Li, N.; Wang, S.; Song, Y.; Liu, Y.; Liu, W. S-1 chemotherapy and intensity-modulated radiotherapy after D1/D2 lymph node dissection in patients with node-positive gastric cancer: A phase I/II study. Br. J. Cancer 2018, 118, 338-343. [CrossRef]

42. Moningi, S.; Ajani, J.A.; Badgwell, B.D.; Murphy, M.B.; Ikoma, N.; Mansfield, P.F.; Ho, J.C.; Suh, Y.; Crane, C.; Herman, J.M. IMRT reduces acute toxicity in patients treated with preoperative chemoradiation for gastric cancer. Adv. Radiat. Oncol. 2019, 5, 369-376. [CrossRef]

43. Wang, J.; Tian, Y.; Tang, Y.; Wang, X.; Li, N.; Ren, H.; Fang, H.; Feng, Y.; Wang, S.; Song, Y. A phase II prospective nonrandomized trial of magnetic resonance imaging-guided hematopoietic bone marrow-sparing radiotherapy for gastric cancer patients with concurrent chemotherapy. Onco Targets Ther. 2016, 9, 2701. [CrossRef] [PubMed]

44. Leone, P.; Vacca, A.; Dammacco, F.; Racanelli, V. Common variable immunodeficiency and gastric malignancies. Int. J. Mol. Sci. 2018, 19, 451. [CrossRef] [PubMed]

45. Yap, Y.L.; So, J.B. Gastric adenocarcinoma occurring in a young patient with common variable immunodeficiency syndrome. Singap. Med. J. 2009, 50, e201-e203.

46. Wasserstein, R.L.; Lazar, N.A. The ASA statement on $p$-values: Context, process, and purpose. Am. Stat. 2016, 70, 129-133. [CrossRef]

47. Basta, Y.L.; Baur, O.L.; van Dieren, S.; Klinkenbijl, J.H.; Fockens, P.; Tytgat, K.M. Is there a benefit of multidisciplinary cancer team meetings for patients with gastrointestinal malignancies? Ann. Surg. Oncol. 2016, 23, 2430-2437. [CrossRef]

48. Du, C.Z.; Li, J.; Cai, Y.; Sun, Y.S.; Xue, W.C.; Gu, J. Effect of multidisciplinary team treatment on outcomes of patients with gastrointestinal malignancy. World J. Gastroenterol. 2011, 17, 2013-2018. [CrossRef] [PubMed]

49. Oxenberg, J.; Papenfuss, W.; Esemuede, I.; Attwood, K.; Simunovic, M.; Kuvshinoff, B.; Francescutti, V. Multidisciplinary cancer conferences for gastrointestinal malignancies result in measureable treatment changes: A prospective study of 149 consecutive patients. Ann. Surg. Oncol. 2015, 22, 1533-1539. [CrossRef]

50. Quéro, L.; Guillerm, S.; Hennequin, C. Neoadjuvant or adjuvant therapy for gastric cancer. World J. Gastrointest. Oncol. 2015, 7, 102-110. [CrossRef]

51. Bang, Y.J.; Van Cutsem, E.; Feyereislova, A.; Chung, H.C.; Shen, L.; Sawaki, A.; Lordick, F.; Ohtsu, A.; Omuro, Y.; Satoh, T.; et al. Trastuzumab in combination with chemotherapy versus chemotherapy alone for treatment of HER2-positive advanced gastric or gastro-oesophageal junction cancer (ToGA): A phase 3, open-label, randomised controlled trial. Lancet (London, England) 2010, 376, 687-697. [CrossRef] 
52. Fuchs, C.S.; Tomasek, J.; Yong, C.J.; Dumitru, F.; Passalacqua, R.; Goswami, C.; Safran, H.; Dos Santos, L.V.; Aprile, G.; Ferry, D.R.; et al. Ramucirumab monotherapy for previously treated advanced gastric or gastro-oesophageal junction adenocarcinoma (REGARD): An international, randomised, multicentre, placebo-controlled, phase 3 trial. Lancet (London, England) 2014, 383, 31-39. [CrossRef]

53. Wilke, H.; Muro, K.; Van Cutsem, E.; Oh, S.C.; Bodoky, G.; Shimada, Y.; Hironaka, S.; Sugimoto, N.; Lipatov, O.; Kim, T.Y.; et al. Ramucirumab plus paclitaxel versus placebo plus paclitaxel in patients with previously treated advanced gastric or gastro-oesophageal junction adenocarcinoma (RAINBOW): A double-blind, randomised phase 3 trial. Lancet Oncol. 2014, 15, 1224-1235. [CrossRef]

54. Wei, L.; Sun, J.; Zhang, N.; Zheng, Y.; Wang, X.; Lv, L.; Liu, J.; Xu, Y.; Shen, Y.; Yang, M. Noncoding RNAs in gastric cancer: Implications for drug resistance. Mol. Cancer 2020, 19, 1-17. [CrossRef]

55. Argentiero, A.; De Summa, S.; Di Fonte, R.; Iacobazzi, R.M.; Porcelli, L.; Da Vià, M.; Brunetti, O.; Azzariti, A.; Silvestris, N.; Solimando, A.G. Gene expression comparison between the lymph node-positive and-negative reveals a peculiar immune microenvironment signature and a theranostic role for WNT targeting in pancreatic ductal adenocarcinoma: A pilot study. Cancers 2019, 11, 942. [CrossRef] [PubMed]

56. Eggar, M.; Davey Smith, G. Misleading meta-analysis. Lessons from "an effective, safe, simple" intervention that wasn't. Br. Med. J. 1995, 310, 752-754.

57. Stroup, D.F.; Berlin, J.A.; Morton, S.C.; Olkin, I.; Williamson, G.D.; Rennie, D.; Moher, D.; Becker, B.J.; Sipe, T.A.; Thacker, S.B. Meta-analysis of observational studies in epidemiology: A proposal for reporting. J. Am. Med. Assoc. 2000, 283, 2008-2012. [CrossRef] [PubMed]

58. Shrier, I.; Boivin, J.-F.; Steele, R.J.; Platt, R.W.; Furlan, A.; Kakuma, R.; Brophy, J.; Rossignol, M. Should meta-analyses of interventions include observational studies in addition to randomized controlled trials? A critical examination of underlying principles. Am. J. Epidemiol. 2007, 166, 1203-1209. [CrossRef]

59. Zhou, M.L.; Kang, M.; Li, G.C.; Guo, X.M.; Zhang, Z. Postoperative chemoradiotherapy versus chemotherapy for R0 resected gastric cancer with D2 lymph node dissection: An up-to-date meta-analysis. World J. Surg. Oncol. 2016, 14, 209. [CrossRef]

60. Huang, Y.Y.; Yang, Q.; Zhou, S.W.; Wei, Y.; Chen, Y.X.; Xie, D.R.; Zhang, B. Postoperative chemoradiotherapy versus postoperative chemotherapy for completely resected gastric cancer with D2 Lymphadenectomy: A meta-analysis. PLoS ONE 2013, 8, e68939.

61. Ohri, N.; Garg, M.K.; Aparo, S.; Kaubisch, A.; Tome, W.; Kennedy, T.J.; Kalnicki, S.; Guha, C. Who benefits from adjuvant radiation therapy for gastric cancer? A meta-analysis. Int. J. Radiat. Biol. Phys. 2013, 86, 330-335. [CrossRef]

62. The Newcastle-Ottawa Scale (NOS) for Assessing the Quality of Nonrandomised Studies in Meta-Analyses. Available online: http://www.ohri.ca/programs/clinical_epidemiology/oxford.asp (accessed on 3 February 2020).

63. Borenstein, M.; Hedges, L.V.; Higgins, J.P.; Rothstein, H.R. Fixed-Effect Versus Random-Effects Models. In Introduction to Meta-Analysis; John Wiley \& Sons: Hoboken, NJ, USA, 2011.

64. Higgins, J.P.T.; Thompson, S.G. Quantifying heterogeneity in a meta-analysis. Stat. Med. 2002, 21, 1539-1558. [CrossRef]

65. Cochran, W.G. The combination of estimates from different experiments. Biometrics 1954, 10, $101-129$. [CrossRef]

66. Higgins, J.P.; Thompson, S.G.; Deeks, J.J.; Altman, D.G. Measuring inconsistency in meta-analyses. Br. Med. J. 2003, 327, 557-560. [CrossRef] [PubMed]

67. Egger, M.; Smith, G.D.; Schneider, M.; Minder, C. Bias in meta-analysis detected by a simple, graphical test. Br. Med. J. 1997, 315, 629-634. [CrossRef]

68. Duval, S.; Tweedie, R. Trim and fill: A simple funnel-plot-based method of testing and adjusting for publication bias in meta-analysis. Biometrics 2000, 56, 455-463. [CrossRef] [PubMed]

69. Declaration of Helsinki: Recommendations Guiding Medical Doctors in Biomedical Research Involving Human Subjects. Available online: https:/www.wma.net/what-we-do/medical-ethics/declaration-ofhelsinki/ (accessed on 3 February 2020).

(C) 2020 by the authors. Licensee MDPI, Basel, Switzerland. This article is an open access article distributed under the terms and conditions of the Creative Commons Attribution (CC BY) license (http://creativecommons.org/licenses/by/4.0/). 